\title{
Hepatocellular carcinoma redirects to ketolysis for progression under nutrition deprivation stress
}

\author{
De Huang ${ }^{1,}$, Tingting $\mathrm{Li}^{1,}{ }^{\text {, }}$, Lin Wang ${ }^{1}$, Long Zhang ${ }^{1}$, Ronghui Yan ${ }^{1}, \mathrm{Kui} \mathrm{Li}^{1}$, Songge Xing ${ }^{1,2}$, Gongwei Wu \\ Lan $\mathrm{Hu}^{1}$, Weidong Jia ${ }^{2}$, Sheng-Cai Lin $^{3}$, Chi V Dang ${ }^{4}$, Libing Song ${ }^{5}$, Ping Gao ${ }^{1}$, Huafeng Zhang ${ }^{1}$
}

${ }^{1}$ CAS Key laboratory of Innate Immunity and Chronic Disease, Innovation Center for Cell Signaling Network, School of Life Science, Hefei National Laboratory for Physical Sciences at Microscale, University of Science and Technology of China, Hefei, Anhui 230027, China; ${ }^{2}$ Anhui Key Laboratory of Hepatopancreatobiliary Surgery, Department of General Surgery, Anhui Provincial Hospital, Hefei, Anhui 230001, China; ${ }^{3}$ State Key Laboratory of Cellular Stress Biology, Innovation Center for Cell Signaling Network, School of Life Sciences, Xiamen University, Xiamen, Fujian 361005, China; ${ }^{4}$ Abramson Cancer Center, University of Pennsylvania, Philadelphia, PA, USA; ${ }^{5}$ State Key Laboratory of Oncology in Southern China and Departments of Experimental Research, Sun Yat-sen University Cancer Center, Guangzhou, Guangdong 510060, China

Cancer cells are known for their capacity to rewire metabolic pathways to support survival and proliferation under various stress conditions. Ketone bodies, though produced in the liver, are not consumed in normal adult liver cells. We find here that ketone catabolism or ketolysis is re-activated in hepatocellular carcinoma (HCC) cells under nutrition deprivation conditions. Mechanistically, 3-oxoacid CoA-transferase 1 (OXCT1), a rate-limiting ketolytic enzyme whose expression is suppressed in normal adult liver tissues, is re-induced by serum starvation-triggered mTORC2AKT-SP1 signaling in HCC cells. Moreover, we observe that enhanced ketolysis in HCC is critical for repression of AMPK activation and protects HCC cells from excessive autophagy, thereby enhancing tumor growth. Importantly, analysis of clinical HCC samples reveals that increased OXCT1 expression predicts higher patient mortality. Taken together, we uncover here a novel metabolic adaptation by which nutrition-deprived HCC cells employ ketone bodies for energy supply and cancer progression.

Keywords: ketone bodies; HCC; OXCT1; AKT; AMPK; autophagy

Cell Research (2016) 26:1112-1130. doi:10.1038/cr.2016.109; published online 20 September 2016

\section{Introduction}

Compared with their normal counterparts, cancer cells are metabolically reprogrammed in order to obtain sufficient energy or additional stimuli to support rapid cell growth and proliferation $[1,2]$. While cancer cells are known to consume glucose, glutamine and fatty acids disproportionately for energy as well as carbon and nitrogen sources for anabolism, nutrient limitation often

*These two authors contributed equally to this work.

Correspondence: Huafeng Zhang ${ }^{\mathrm{a}}$, Ping Gao ${ }^{\mathrm{b}}$, Libing Song ${ }^{\mathrm{c}}$

aE-mail: hzhang22@ustc.edu.cn

bE-mail: pgao2@ustc.edu.cn

${ }^{\mathrm{c} E}$-mail: songlb@sysucc.org.cn

Received 1 July 2016; revised 10 July 2016; accepted 14 July 2016; pub-

lished online 20 September 2016 occurs during tumor development. Increasing evidence has demonstrated that cancer cells are widely open to additional nutrient sources under nutrition-limiting conditions [3]. Two groups reported recently that a variety of cancer types consume acetate avidly to fuel cancer growth [4-6]. More recently, Loo et al. [7] documented that metastatic colorectal cancer cells rely on extracellular metabolite creatine to facilitate metastasis and cancer progression. Moreover, in addition to early reports that established lactate recycling as a significant fuel source for cancer progression $[8,9]$, a most recent study showed that accumulated lactate facilitates hypoxia signaling to stimulate cancer growth [10]. Together, these significant findings all point to an alarming possibility that multiple seemingly wasteful metabolites are fuels for cancer cells, leaving it wide open for exploring potential alternative nutrients for tumors to shape up a comprehensive picture 
on cancer metabolism.

Ketone bodies comprise three distinct small molecules: acetone, acetoacetate (AcAc) and $\beta$-hydroxybutyrate $(\beta-\mathrm{HB})$. The production of ketone bodies, or ketogenesis, mainly occurs in the liver where acetyl-CoA is first converted to AcAc, which is further catalyzed by $\beta$-hydroxybutyrate dehydrogenase (BDH1) to produce $\beta$-HB. While acetone is unable to be further metabolized to produce ATP, both AcAc and -HB are exported into bloodstream and consumed by extra-hepatic tissues, such as muscle, kidney or brain [11]. Of note, ketone bodies are also able to pass through the blood-brain barrier, entering the brain to serve as a major energy source during starvation [12]. For ketone body catabolism, or ketolysis, $\beta-\mathrm{HB}$ is oxidized back to AcAc also by BDH1. Next, succinyl-CoA donates its CoA to AcAc to form acetoacetyl-CoA (AcAc-CoA), a rate-limiting reaction catalyzed by 3-oxoacid CoA-transferase 1 (OXCT1, also known as SCOT). Acetyl-CoA acetyltransferase 1 (ACAT1) then converts AcAc-CoA to two acetyl-CoAs, which feed into tricarboxylic acid (TCA) cycle for ATP production [13]. For the whole organism, ketone bodies provide a fast and efficient way for energy supply during starvation, which links dietary lipids or adipose triglycerides to the TCA cycle and respiratory chain. As a key enzyme of ketolysis, OXCT1 is expressed abundantly in heart, brain and kidney; however, its expression is found to be repressed in adult liver and liver cell lines [14, 15], largely considered as a mechanism to prevent a futile cycle of ketone body synthesis and catabolism in the same tissue. Hence, while it is well established that the liver is the major factory for ketone body production, up to date, there is no documentation showing that adult human liver cells could utilize ketone bodies as energy sources, at least to the best of our knowledge.

Recently, we investigated the metabolic adaptation of human hepatocellular carcinoma (HCC) cells under various stress conditions [16-18]. We have uncovered cMyc-mediated activation of serine synthesis pathway when glucose or glutamine is deprived [18] and HIF-1-mediated suppression of fatty acid $\beta$-oxidation when oxygen is scarce [16]. Further screening for changes of lipid metabolism-related genes led us to a surprising observation that a major enzyme for ketolysis, OXCT1, is dramatically induced in nutrition-deprived HCC cells. OXCT1 is known to be abundantly expressed in embryonic livers; however, its expression disappears in the adult liver, which has been suggested as the major reason for the absence of consumption of ketone bodies in adult livers [19]. Hence, the identification of high expression of OXCT1 in HCC cells led us to hypothesize that liver cancer cells, like that of fetal livers or the brain, might be able to use ketone bodies to fuel cell growth under nutrition-limiting conditions which, in fact, are unavoidable circumstances during cancer development. In this study, our results established that nutrition starvation promotes metabolic reprogramming of HCC cells by stimulating OXCT1 expression and consequent ketone body utilization for energy supply. Further exploration revealed that ketone body-derived ATP production diminished AMPK phosphorylation and autophagy during nutrition deprivation. Collectively, OXCT1 activation in liver cancer cells facilitates ketone body utilization as a self-serving fuel source for cell growth under nutrition limitation. These findings open a way for treating HCC patients by harnessing the dysregulated ketone body metabolism.

\section{Results}

Ketolysis is re-activated in nutrition-starved HCC cells to facilitate cell proliferation

Our recent work showed that fatty acid $\beta$-oxidation in cancer cells is inhibited to ease oxidative stress and to activate proliferative signaling under hypoxic microenvironment [16]. We further investigated lipid metabolic changes under specific nutrition-limiting conditions. We first cultured human HepG2 cells under glucose-, glutamine- or serum-starved conditions and performed reverse transcription and quantitative real-time PCR (qRT-PCR) to analyze the expression of lipid metabolism-related genes. Interestingly, we found that several major genes responsible for both ketogenesis and ketolysis are markedly altered by serum starvation (Figure 1A). While it was not surprising that expression of genes related to ketogenesis, such as BDH1 and HMG-CoA synthase 2 (HMGCS2), was activated in liver cells in response to starvation $[20,21]$, it was unexpected that OXCT1, which encodes the key enzyme of ketolysis, was significantly expressed in HepG2 cells under serum starvation compared with normal culture conditions (Figure 1A). Consistent with qRT-PCR results, depletion of serum, but not glucose or glutamine, markedly stimulated OXCT1 protein expression in various $\mathrm{HCC}$ cell lines, including HepG2, Hep3B and PLC cells (Figure 1B and Supplementary information, Figure S1A). BDH1, which catalyzes reversible reaction in both ketogenesis and ketolysis, was also upregulated under serum starvation conditions (Figure 1A, 1B and Supplementary information, Figure S1A). We also observed that serum starvation elevated the protein levels of OXCT1 and BDH1 in a time-dependent manner in HepG2, Hep3B and PLC cells (Figure 1C and Supplementary information, Figure S1B), but not in an untransformed liver cell line THLE3 [22] at either protein or mRNA levels (Figure 1C and 
A

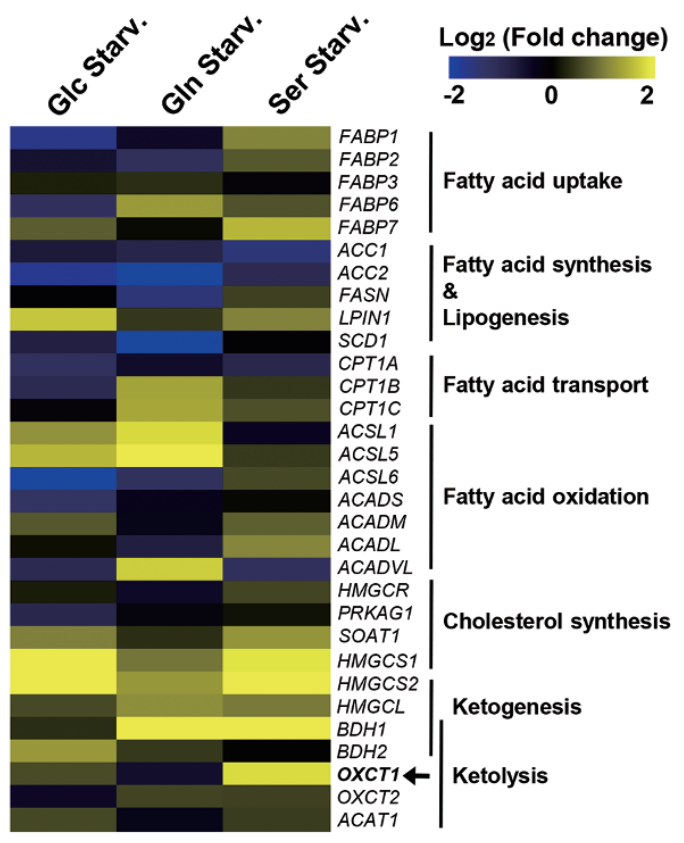

B

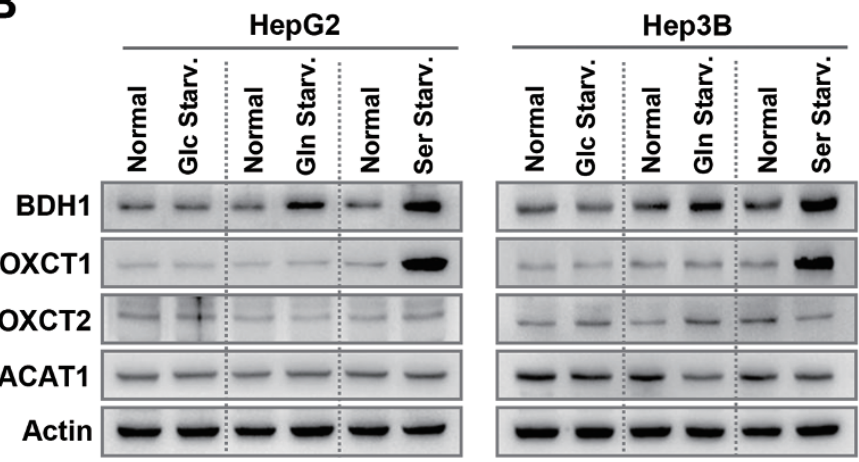

C

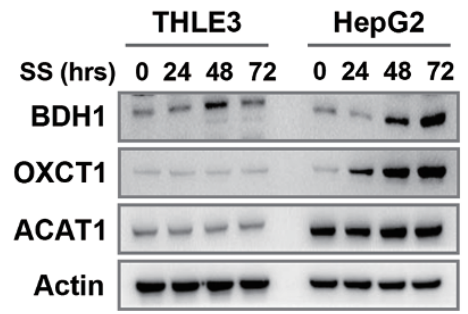

E

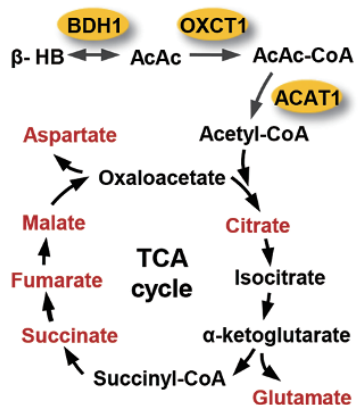

D
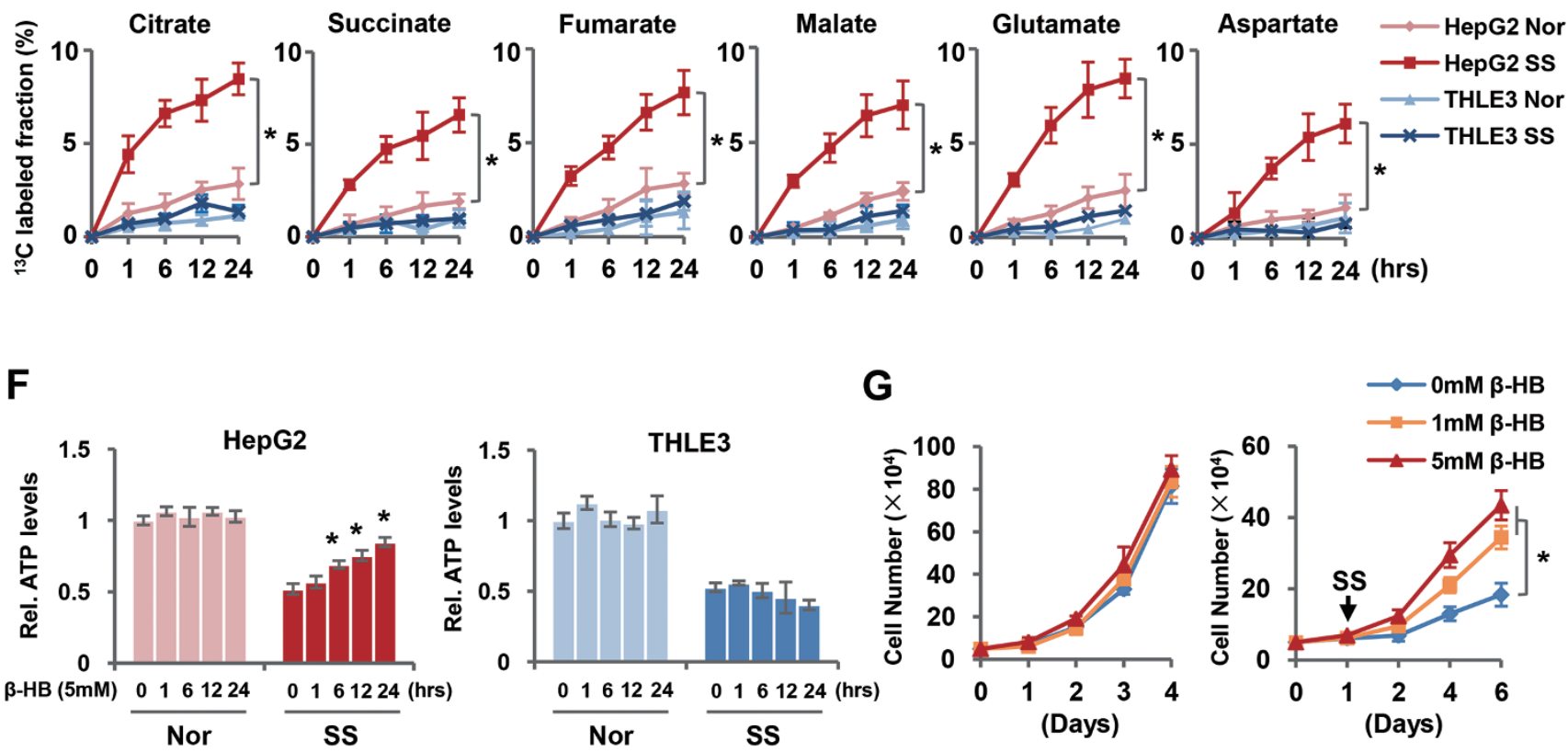

Supplementary information, Figure S1C), indicating that serum starvation specifically activates OXCT1 and BDH1 expression in liver cancer cells.

Induction of expression of both $\mathrm{BDH} 1$ and OXCT1 by serum deprivation, two key enzymes for $\beta$-HB catabolism in mitochondria, suggested the activation of ketolysis in serum-starved liver cancer cells. Thus, we traced the metabolic flux of ketone body using ${ }^{13} \mathrm{C}$-labeled $[2$, $\left.{ }^{4}-{ }^{13} \mathrm{C}_{2}\right] \beta$-HB by gas-chromatography-mass-spectrometry (GC-MS) analysis. Our results revealed that serum-starved HepG2 cells rapidly acquired the capacity of catabolizing ${ }^{13} \mathrm{C}$-labeled $\beta$-HB into various TCA cycle metabolites such as citrate, succinate, fumarate, malate, glutamate and aspartate, while non-serum-starved HepG2 
Figure 1 Ketolysis is re-activated in nutrition-starved HCC cells to facilitate cell proliferation. (A) Heat map from qRT-PCR analysis showed the mRNA expression of lipid metabolism-related genes in HepG2 cells cultured under glucose or glutamine or serum starvation for $24 \mathrm{~h}$ as compared with normal culture condition. Yellow indicates upregulated genes whereas blue indicates downregulated genes. (B) Western blot analysis of BDH1, OXCT1/2 and ACAT1 expression in HepG2 and Hep3B cells cultured under normal, glucose or glutamine or serum starvation conditions for $48 \mathrm{~h}$. (C) Western blot analysis of BDH1, OXCT1 and ACAT1 expression in THLE3 and HepG2 cells cultured under normal $(0 \mathrm{~h})$ or serum starvation (SS) conditions for the indicated hours. (D) GC-MS analysis of ${ }^{13} \mathrm{C}$-labeled metabolites in HepG2 and THLE3 cells cultured under normal (Nor) or serum starvation (SS) conditions for $48 \mathrm{~h}$ followed by incubating with $5 \mathrm{mM}\left[2,4-{ }^{13} \mathrm{C}_{2}\right] \beta-\mathrm{HB}$ for $0,1,6,12$ and $24 \mathrm{~h}$. Data were presented as mean $\pm \mathrm{SD}$. ${ }^{*} P<0.05$ as compared between the indicated groups. (E) Schematic diagram of metabolic flux from ketolysis to TCA cycle, in which metabolites with $\left[2,4-{ }^{13} \mathrm{C}_{2}\right] \beta$-HB-derived carbons measured in $\mathbf{D}$ were marked in red. (F) Cellular ATP levels were measured in HepG2 and THLE3 cells cultured under normal (Nor) or SS conditions for $48 \mathrm{~h}$ followed by incubating with $5 \mathrm{mM}$ exogenous $\beta$-HB for $0,1,6,12$ and $24 \mathrm{~h}$. Values were normalized to cellular protein. Data were presented as mean \pm SD. ${ }^{*} P<0.05$ as compared with Nor group with $0 \mathrm{~h} \beta$-HB incubation. (G) Growth curves of HepG2 cells cultured in the presence or absence of $1 \mathrm{mM}$ or $5 \mathrm{mM} \beta$-HB under normal (left panel) or SS (right panel) conditions. Arrow indicates the time point that SS started. Cell numbers were determined by trypan blue counting. Data were presented as mean \pm SD. ${ }^{*} P<0.05$ as compared between the indicated groups.

cells hardly utilized $\beta$-HB (Figure 1D). Similar results were observed in Hep3B and PLC cells (Supplementary information, Figure S1D) and the steady state labeling of TCA metabolites by ${ }^{13} \mathrm{C}$-labeled $\beta$-HB occurred between 24 and $48 \mathrm{~h}$ (data not shown). Consistent with previous reports $[14,19]$, normal THLE3 liver cells lack the ability to metabolize $\beta$-HB under either condition (Figure 1D and Supplementary information, Figure S1E). Intriguingly, serum-starved HepG2 cells catabolized more $\beta$-HB when glucose and glutamine were simultaneously reduced in culture medium (Supplementary information, Figure S1E). These results using metabolic tracing of ${ }^{13} \mathrm{C}$-labeled $\beta$-HB collectively suggest an increased fraction of $\beta$-HB-derived acetyl-CoA entering TCA cycle to produce ATP under serum deprivation in HCC cells (Figure $1 \mathrm{E}$ ). Indeed, supplementation of exogenous $\beta$-HB gradually recovered cellular ATP levels in serum-starved HepG2 cells but not in THLE3 cells (Figure 1F). Furthermore, while additional $\beta$-HB had no effect on proliferation of HepG2 cells in normal culture conditions, it promoted proliferation of serum-starved HepG2 cells significantly (Figure 1G), demonstrating that HCC cells adapt to nutrition depletion stress by using $\beta-\mathrm{HB}$ as an energy source to facilitate cell growth.

OXCT1 is critical for induction of ketolysis in nutrition-starved HCC cells

Next, we sought to determine which enzyme(s) is critically responsible for nutrition starvation-induced ketone body catabolism in HCC cells. We first generated stable HepG2 cell lines, in which BDH1 or OXCT1 expression was knocked down with specific shRNAs (Figure 2A and Supplementary information, Figure S2A) and cultured the cells with $\left[2,4-{ }^{13} \mathrm{C}_{2}\right] \beta-\mathrm{HB}$. GC-MS analysis showed that knockdown of BDH1 or OXCT1 diminished serum starvation-stimulated conversation of $\left[2,4-{ }^{13} \mathrm{C}_{2}\right]$ $\beta$-HB into TCA cycle metabolites in HepG2 cells (Figure 2A and Supplementary information, Figure S2A), demonstrating that both $\mathrm{BDH} 1$ and $\mathrm{OXCT} 1$ are required for ketolysis of $\beta$-HB under stress conditions. On the other hand, forced expression of BDH1 or OXCT1 in HepG2 cells dramatically reduced the concentration of supplemented $\beta$-HB in culture medium (Figure 2B), suggesting that both $\mathrm{BDH} 1$ and $\mathrm{OXCT} 1$ promoted cellular uptake of $\beta$-HB. Further analysis showed that forced expression of BDH1 increased, while OXCT1 decreased, intracellular AcAc level in HepG2 cells, confirming that BDH1 and OXCT1 sequentially catalyze the consumption of $\beta$-HB (Figure 2C). However, it is very intriguing to observe that only overexpression of OXCT1, but not $\mathrm{BDH} 1$, markedly promoted catabolism of $\left[2,4-{ }^{13} \mathrm{C}_{2}\right]$ $\beta$-HB into TCA cycle metabolites in HepG2, Hep3B and THLE3 cells (Figure 2D, 2E and Supplementary information, Figure S2B). Considering that OXCT1 is absent while BDH1 is abundantly present in normal liver tissues (Supplementary information, Figure S2C), or under normal culture [14], we concluded that it was the induction of OXCT1 expression, but not that of BDH1 expression, that was responsible for the reactivation of ketolysis in serum-starved HCC cells, albeit both enzymes catalyzing rate-limiting steps of ketolysis. Hence, we hereafter focused our study on OXCT1.

Most ketogenesis occurs in the mitochondria of liver cells where acetyl-CoA is sequentially catalyzed by HMGCS2 and HMGCL to produce AcAc and $\beta$-HB [23]. Indeed, our data showed that protein levels of HMGCS2 were increased in HCC cells (including HepG2 and Hep3B) as well as in normal liver cells (THLE3) under serum starvation (Supplementary information, Figure S2D), suggesting that both normal and HCC cells were 
A

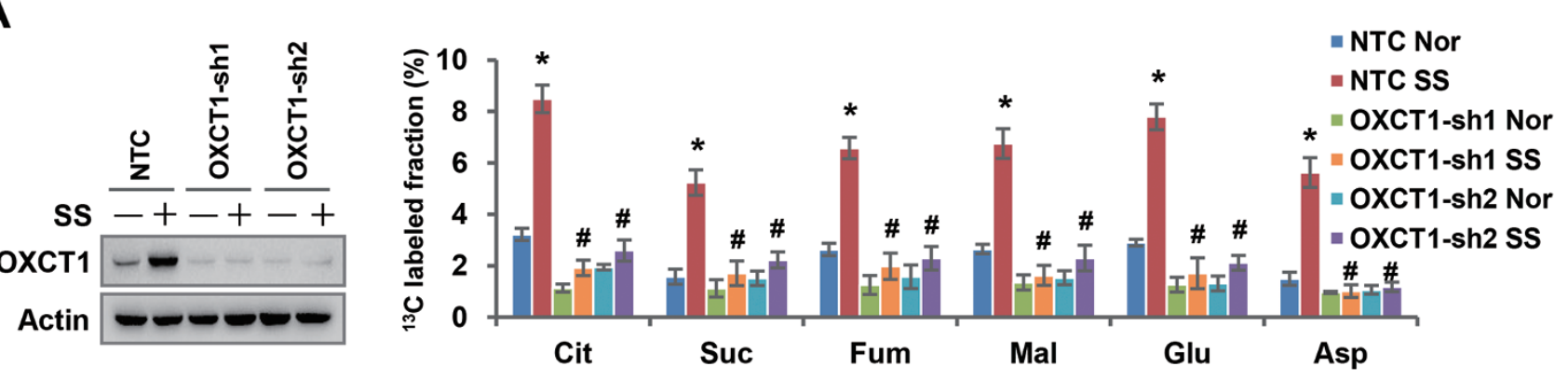

B
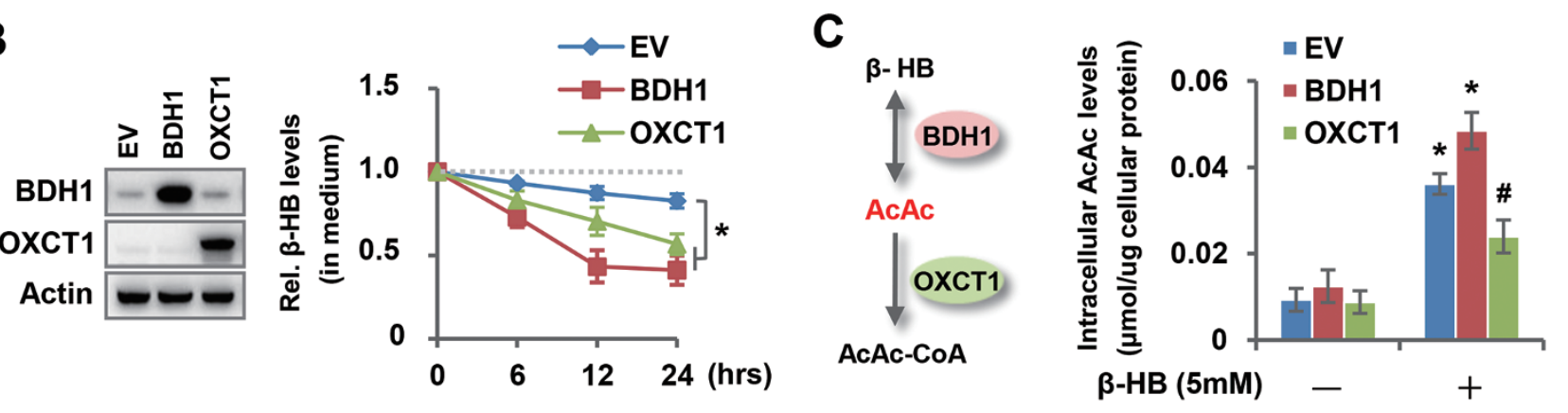

D

HepG2:

E

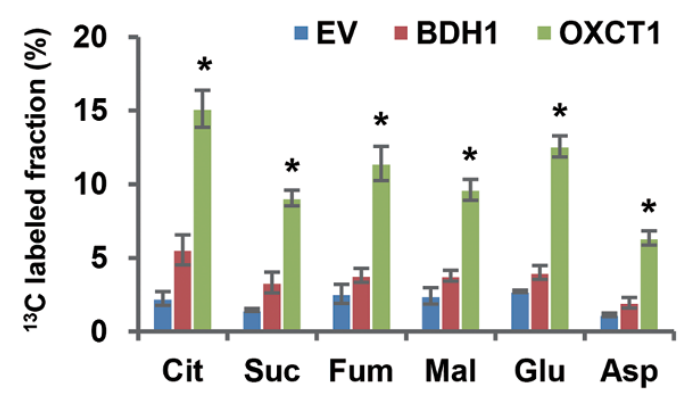

Hep3B:

$\mathbf{F}$

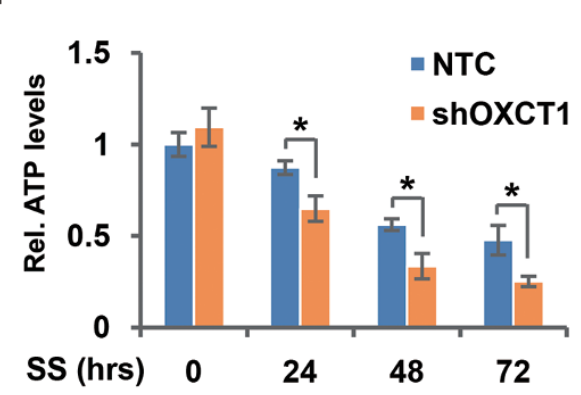

H

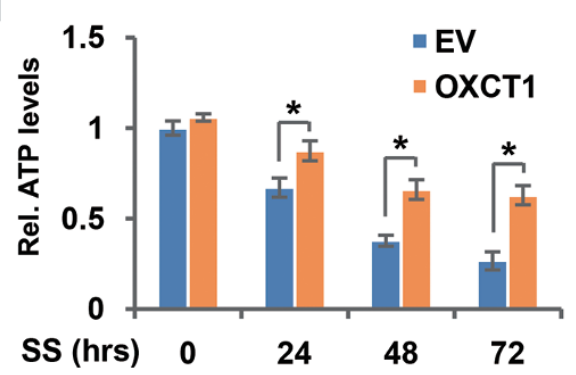

G
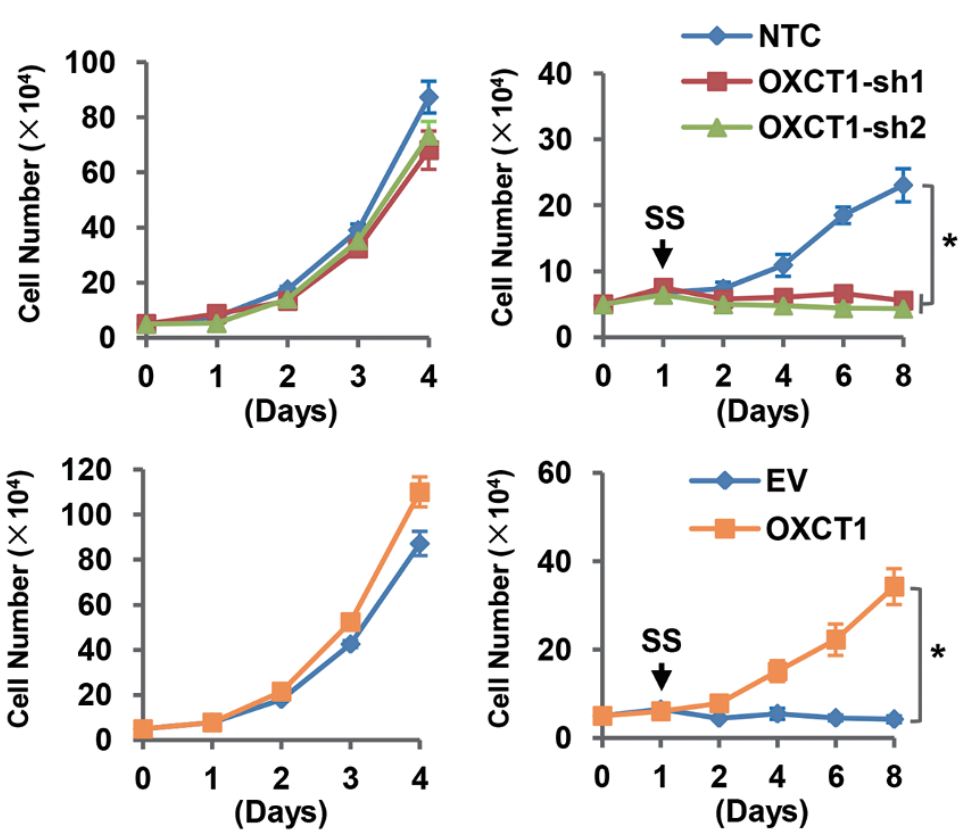

-EV $=\mathrm{BDH} 1 \quad$ - OXCT1

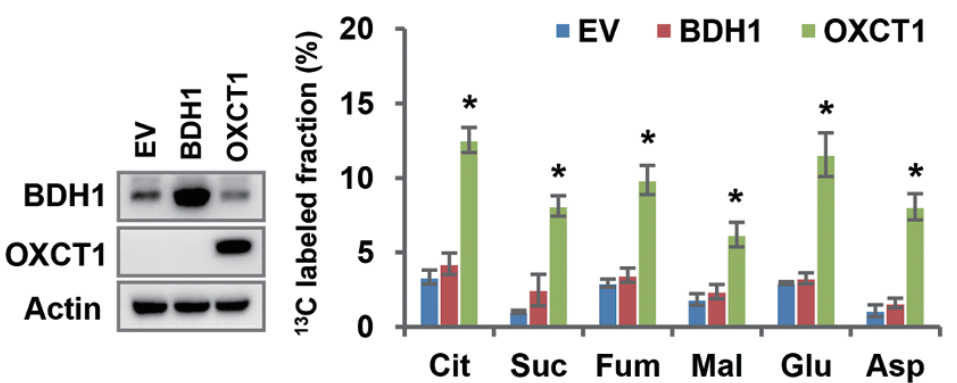


able to generate ketone bodies. Further analysis of cellular $\beta$-HB and AcAc revealed that serum starvation elevated the levels of both ketone bodies in HepG2 cells (Supplementary information, Figure S2E), indicating enhanced ketogenesis under serum starvation in HCC cells. Moreover, knockdown of OXCT1 led to further accumulation of both $\beta$-HB and AcAc under serum starvation (Supplementary information, Figure S2E), demonstrating that ketone bodies produced by HCC cells could be utilized by themselves when OXCT1 expression was induced under nutrition-depleted conditions.

We observed gradually reduced cellular ATP levels by serum starvation in HepG2 cells, which was further reduced upon OXCT1 knockdown (Figure 2F). Consistently, knockdown of OXCT1 by shRNAs had no obvious effect on cell proliferation under normal conditions but completely blocked cell growth in serum-starved HepG2 cells (Figure 2G). More intriguingly, when OXCT1 expression was restored in OXCT1-knockdown HepG2 cells, ATP levels as well as cell growth were significantly recovered under serum starvation (Figure $2 \mathrm{H}$ and 2I). Similar results were also observed in Hep3B cells (Supplementary information, Figure S2F and S2G). As these results were obtained when no exogenous $\beta$-HB was included in the culture medium, we hypothesized that cells with the expression of OXCT1 can use the ketone bodies produced by themselves, as hepatocytes are well-known as the major source of ketone body produc- tion and our results also showed enhanced production of ketone bodies under serum starvation (Supplementary information, Figure S2E). To directly test this hypothesis, we manipulated the expression of HMGCS2, a critical ketogenic enzyme, and the results showed that, when ketone body production was suppressed by knockdown of HMGCS2, OXCT1-stimulated cell proliferation was largely abolished (Supplementary information, Figure $\mathrm{S} 2 \mathrm{H}$ and S2I). Intriguingly, in the presence of exogenous $\beta$-HB, OXCT1 overexpression can still promote cell proliferation in HMGCS2-knockdown HCC cells (Supplementary information, Figure S2I). Taken together, these data demonstrate that OXCT1 expression is critical for maintaining cellular ATP homeostasis via ketolysis to sustain cell proliferation in HCC cells especially under nutrition starvation.

\section{Activation of mTORC2-AKT-SP1 signaling induces OXCT1 expression and ketolysis in nutrition-limiting HCC cells}

We then investigated the mechanism by which serum starvation activates OXCT1 expression and ketolysis in HCC cells. As reported previously [24-27], we observed that phosphorylation levels of AMPK, AKT1 and ERK $1 / 2$ were increased rapidly in response to serum starvation in HepG2 cells (Figure 3A). Consistent with previous reports that serum starvation stimulated NF- $\kappa B$ activity [28], we found accelerated degradation of $\mathrm{I} \kappa \mathrm{B} \alpha$

Figure 2 OXCT1 is critical for induction of ketolysis in nutrition-starved HCC cells. (A) HepG2 cells stably expressing OXCT1 shRNAs or non-targeting control (NTC) were cultured under normal (Nor) or SS conditions for $48 \mathrm{~h}$ followed by incubating with $5 \mathrm{mM}$ of $\left[2,4-{ }^{13} \mathrm{C}_{2}\right] \beta$-HB for $24 \mathrm{~h}$ and subsequent isotope tracing of ${ }^{13} \mathrm{C}$-labeled metabolites by GC-MS. OXCT1 expression was determined by western blot. Data were presented as mean \pm SD. ${ }^{*} P<0.05$ as compared with Nor cultured NTC group; ${ }^{\#} P<0.05$ as compared with SS-treated NTC group. (B) Medium $\beta$-HB was measured in HepG2 cells stably expressing $\mathrm{BDH} 1, \mathrm{OXCT} 1$ or empty vector (EV) after incubating with $5 \mathrm{mM}$ exogenous $\beta$-HB for the indicated hours. OXCT1 and BDH1 expression were determined by western blot. Data were presented as mean \pm SD. ${ }^{*} P<0.05$ as compared between the indicated groups. (C) Intracellular AcAc was measured after incubating with or without 5 mM exogenous $\beta$-HB for $24 \mathrm{~h}$ in HepG2 cells stably expressing BDH1, OXCT1 or EV control. Values were normalized to cellular protein. Data were presented as mean \pm SD. ${ }^{*} P<0.05$ as compared with EV group without $\beta-\mathrm{HB} ;{ }^{\#} P<0.05$ as compared with EV group with $\beta$-HB treatment. (D, E) GC-MS analysis of ${ }^{13} \mathrm{C}$-labeled metabolites after incubating with $5 \mathrm{mM}\left[2,4-{ }^{13} \mathrm{C}_{2}\right] \beta-\mathrm{HB}$ for $24 \mathrm{~h}$ in HepG2 (D) and Hep3B (E) cells stably expressing BDH1, OXCT1 or EV. BDH1 and OXCT1 expression in Hep3B cells were determined by western blot. Data were presented as mean \pm SD. ${ }^{*} P<0.05$ as compared with corresponding EV group. (F) Cellular ATP levels were measured in HepG2 cells stably expressing OXCT1 shRNAs or NTC after SS treatment for the indicated hours. Values were normalized to cellular protein. Data were presented as mean \pm SD. ${ }^{*} P<0.05$ as compared between the indicated groups. (G) Growth curves of HepG2 cells stably expressing OXCT1 shRNAs (sh1 and sh2) or NTC cultured under normal or SS conditions. Arrow indicates the time point that SS started. Cell numbers were determined by trypan blue counting. Data were presented as mean $\pm \mathrm{SD}$. ${ }^{*} P<0.05$ as compared between the indicated groups. $(\mathrm{H}) \mathrm{HepG} 2$ cells stably expressing OXCT1 shRNA (targeting 3'UTR) were further infected with viruses expressing pSIN-3×flag-OXCT1 or pSIN-EV, followed by cellular ATP measurement after serum starvation (SS) treatment for the indicated hours. Values were normalized to cellular protein. Data were presented as mean $\pm \mathrm{SD}$. ${ }^{*} P<0.05$ as compared between the indicated groups. (I) HepG2 cells stably expressing OXCT1 shRNA (targeting 3'UTR) were further infected with viruses expressing pSIN-3×flag-OXCT1 or pSIN-EV. Cells were treated with or without SS starting from arrow-indicated day. Cell numbers were determined by trypan blue counting. Data were presented as mean $\pm \mathrm{SD}$. ${ }^{*} P<0.05$ as compared between the indicated group. 


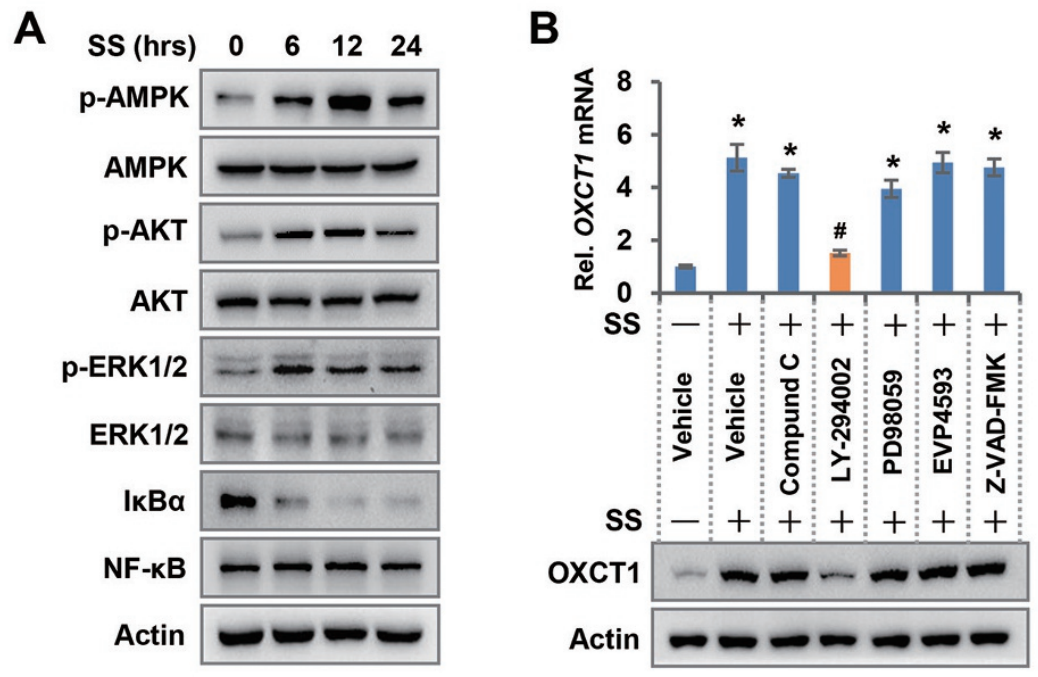

C

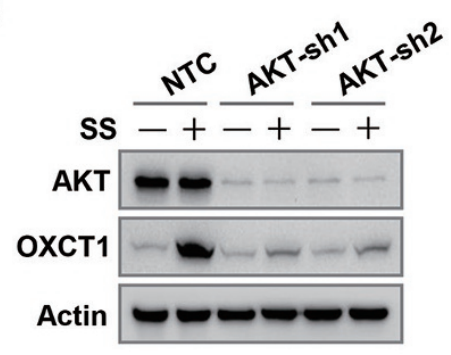

$\mathbf{L}$

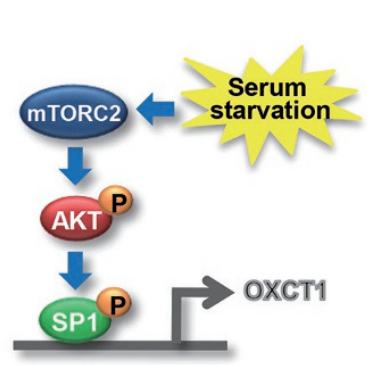

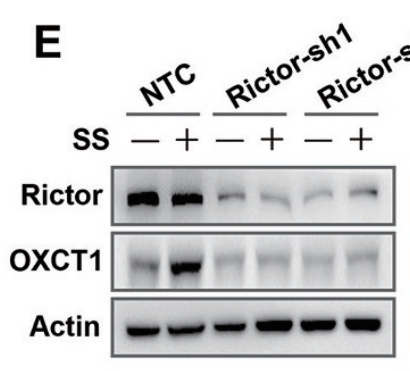
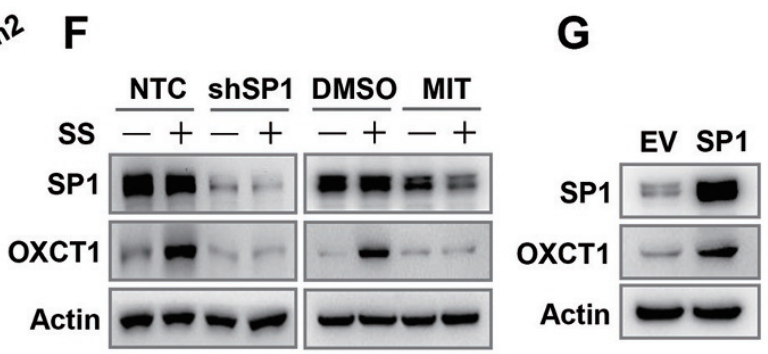

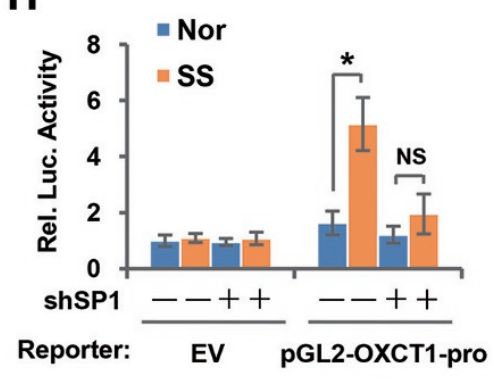

K

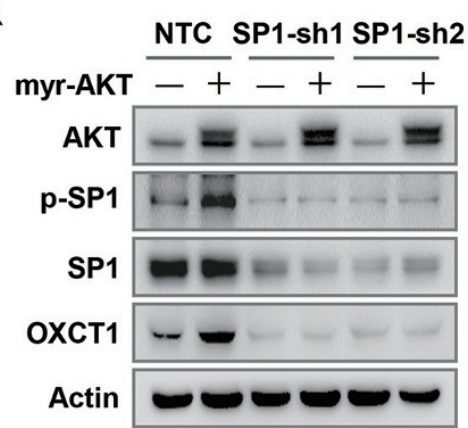

H
M

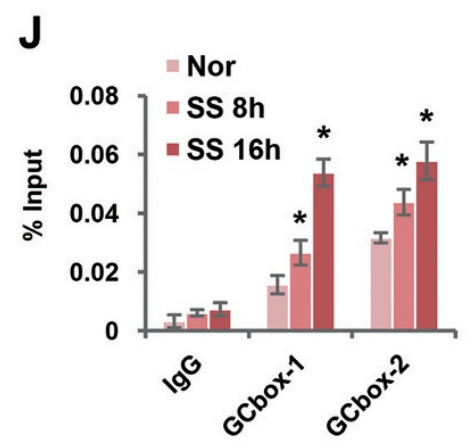

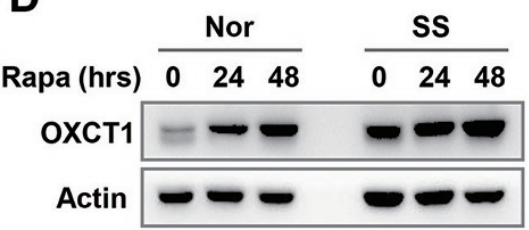
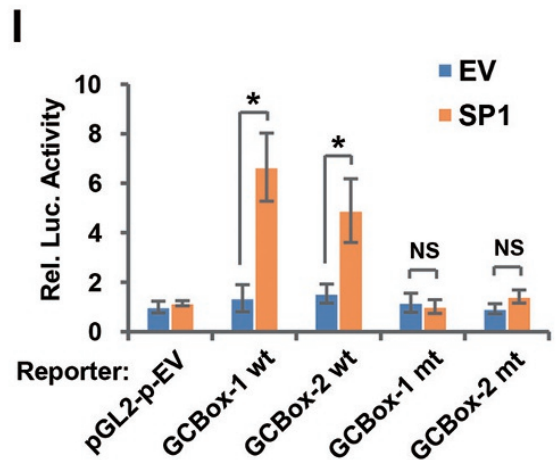

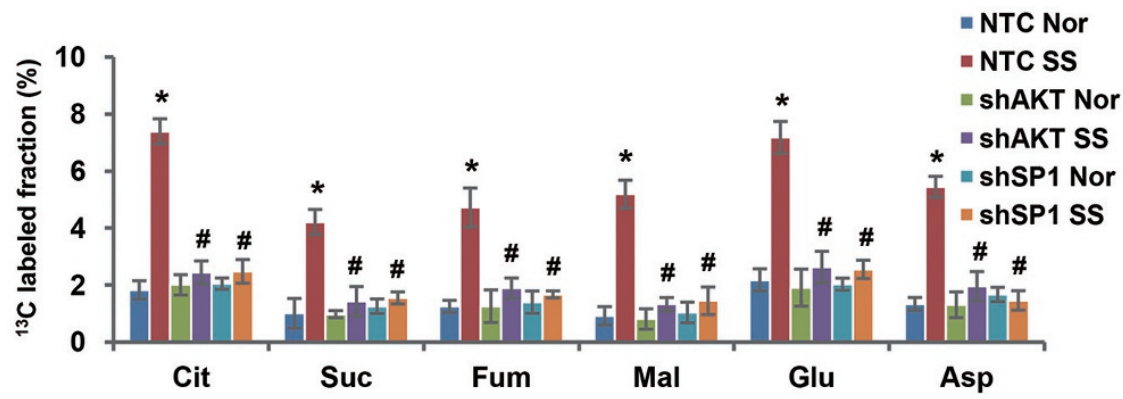


Figure 3 Activation of mTORC2-AKT-SP1 signaling induces OXCT1 expression and ketolysis in nutrition-limiting HCC cells. (A) Western blot analysis of p-AMPK (threonine 172 phosphorylation of AMPK), AMPK, p-AKT (serine 473 phosphorylation

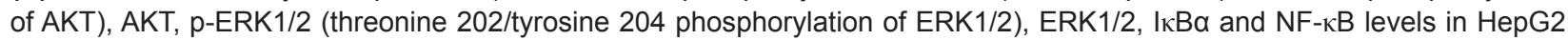
cells under normal $(0 \mathrm{~h})$ or serum starvation conditions for 6, 12 and $24 \mathrm{~h}$. (B) qRT-PCR and western blot analysis of OXCT1 expression in HepG2 cells incubated with vehicle (DMSO), compound C (10 $\mu \mathrm{M})$, LY-294002 (50 $\mu \mathrm{M})$, PD98059 (50 $\mu \mathrm{M})$, EVP4593 $(1 \mu \mathrm{M})$ or Z-VAD-FMK $(50 \mu \mathrm{M})$ for $2 \mathrm{~h}$ prior to SS for 24 or $48 \mathrm{~h}$. Data were presented as mean \pm SD. ${ }^{*} P<0.05$ as compared with vehicle-treated normal group; ${ }^{*} P<0.05$ as compared with vehicle-treated SS group. (C) AKT and OXCT1 protein levels were determined by western blot in HepG2 cells stably expressing AKT shRNAs (sh1 and sh2) or NTC under normal or SS conditions for 48 h. (D) Western blot analysis of OXCT1 expression in HepG2 cells treated with vehicle (DMSO) or $50 \mathrm{nM}$ rapamycin for 24 and $48 \mathrm{~h}$ in the presence or absence of serum. (E) HepG2 cells stably expressing Rictor shRNAs or NTC were cultured under Nor or SS conditions for $48 \mathrm{~h}$. Rictor and OXCT1 expression were determined by western blot. (F) Western blot analysis of SP1 and OXCT1 expression in HepG2 cells expressing SP1 shRNA or NTC, or in HepG2 cells pretreated with vehicle (DMSO) or MIT $(20 \mathrm{nM})$ for $2 \mathrm{~h}$, under normal or SS conditions for $48 \mathrm{~h}$. (G) Western blot analysis of SP1 and OXCT1 levels in HepG2 cells expressing pSIN-SP1 or pSIN-EV. (H) HEK293 cells were co-transfected with SP1 shRNAs and pGL2-OXCT1-promoter or pGL2-EV luciferase reporter vectors, and $24 \mathrm{~h}$ after transfection, medium was changed to normal (Nor) or SS for another $24 \mathrm{~h}$, followed by dual luciferase assay. Data were presented as mean \pm SD. ${ }^{*} P<0.05$ as compared between the indicated groups. (I) HEK293 cells were co-transfected with pSIN-SP1 and pGL2-P-GCbox (GCbox-1/2 wt), pGL2-P-GCbox mutants (GCbox-1/2 mt) or pGL2-P-EV luciferase vectors. Dual luciferase assay were performed $24 \mathrm{~h}$ after transfection. Data were presented as mean \pm SD. ${ }^{*} P<0.05$ as compared between the indicated groups. (J) The occupancy of potential GC-box (GCBox-1/2) by SP1 was analyzed by ChIP assay in HepG2 cells cultured under normal (Nor) or SS conditions for the indicated hours using anti-SP1 antibody or lgG control. Data were presented as mean \pm SD. ${ }^{*} P<0.05$ as compared with corresponding Nor group. (K) HepG2 cells stably expressing SP1 shRNAs or NTC were further infected with viruses expressing pBaBe-myr-flag-AKT (myr-AKT) or pBaBe-EV, followed by western blot analysis of AKT1, p-SP1 (threonine 453 phosphorylation of SP1), SP1 and OXCT1 levels. (L) Schematic representation for serum starvation-activated OXCT1 transcription mediated by mTORC2, AKT and SP1. (M) HepG2 cells stably expressing AKT shRNA, SP1 shRNA or NTC were cultured under Nor or SS conditions for $48 \mathrm{~h}$ followed by incubating with $5 \mathrm{mM}$ of $\left[2,4-{ }^{13} \mathrm{C}_{2}\right] \beta-\mathrm{HB}$ for $24 \mathrm{~h}$ and subsequent isotope tracing of ${ }^{13} \mathrm{C}$-labeled metabolites by GC-MS. Data were presented as mean $\pm \mathrm{SD}$. ${ }^{*} P<0.05$ as compared with Nor-treated NTC group; ${ }^{\#} P<0.05$ as compared with SS-treated NTC group.

in serum-starved HepG2 cells, indicating potential NF$\kappa \mathrm{B}$ activation (Figure $3 \mathrm{~A}$ ). To identify the pathways that might be involved in serum starvation-induced OXCT1 expression, we treated HepG2 cells with various inhibitors under serum deprivation conditions, including compound C (an AMPK inhibitor), LY-294002 (a PI3K/ AKT inhibitor), PD98059 (an inhibitor of MEK which is an upstream effecter of Erk-1/2), EVP4593 (an NF-kB inhibitor) and Z-VAD-FMK (an apoptosis inhibitor). Surprisingly, only LY-294002 remarkably attenuated the serum starvation-induced mRNA and protein expression of OXCT1 (Figure 3B). Furthermore, knockdown of AKT by specific shRNAs blocked the activation of OXCT1 at both mRNA (Supplementary information, Figure S3A) and protein (Figure 3C) levels under serum starvation conditions in HepG2 cells, demonstrating that AKT is involved in the activation of OXCT1 in nutrition-limiting HCC cells.

Since mTORC2 was reported to stimulate AKT phosphorylation at serine 473 [29], we treated HepG2 cells with rapamycin to activate mTORC2. The results revealed that OXCT1 expression level was increased by treatment with rapamycin in HepG2 cells (Figure 3D). Knockdown of Rictor, an essential component of the mTORC2 complex, abolished the induction of AKT phosphorylation at serine 473 (Supplementary information, Figure S3B) and OXCT1 expression (Figure 3E) by serum starvation, further demonstrating that mTORC2 acts upstream of AKT phosphorylation and OXCT1 induction under serum starvation.

A previous report showed that the promoter region of OXCT1 harbors two GC-box sequences which are potential binding sites of transcription factor SP1 [15]; however, the precise contribution of these GC-box sequences to $O X C T 1$ transcription has not been fully explored. Our western blot and qRT-PCR analysis revealed that knockdown of SP1 by shRNAs or blocking SP1 transactivity by mithramycin A (MIT), a specific SP1 inhibitor, significantly decreased OXCT1 expression level in serum-starved HepG2 cells (Figure 3F and Supplementary information, Figure S3C). Furthermore, forced expression of SP1 enhanced OXCT1 expression in HepG2 cells (Figure 3G), suggesting that SP1 is involved in OXCT1 regulation. Next, we inserted the $O X C T 1$ promoter region or GC-box fragments (Supplementary information, Figure S3D) into reporter plasmids and performed dual luciferase analysis. Serum starvation significantly enhanced the luciferase activity of $O X C T 1$ promoter re- 
porter, which was attenuated by SP1 knockdown (Figure $3 \mathrm{H})$. Furthermore, forced expression of SP1 enhanced the activity of the two luciferase reporters containing the wild-type (wt) GC-box but not the ones containing the mutant (mt) GC-box (Figure 3I), suggesting that SP1 stimulates $O X C T 1$ expression by targeting these $\mathrm{GC}$ box sequences in the promoter regions of $O X C T 1$ under serum starvation. ChIP assay further confirmed that SP1
A

B

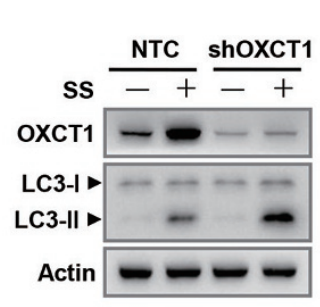

E

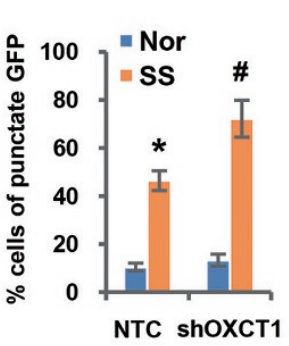

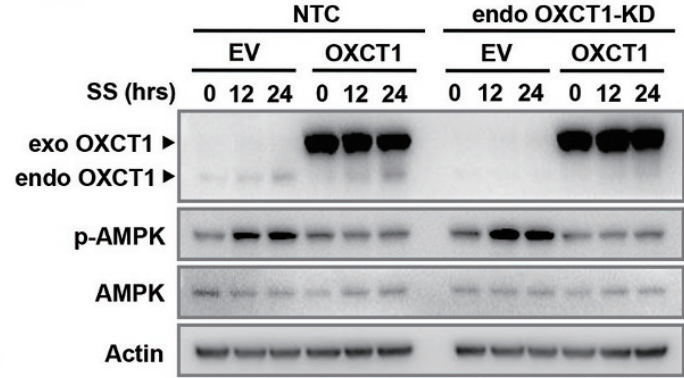

C

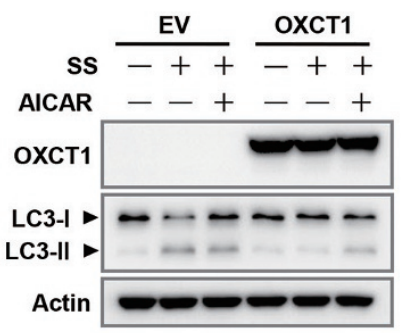

F

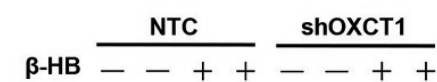

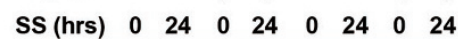

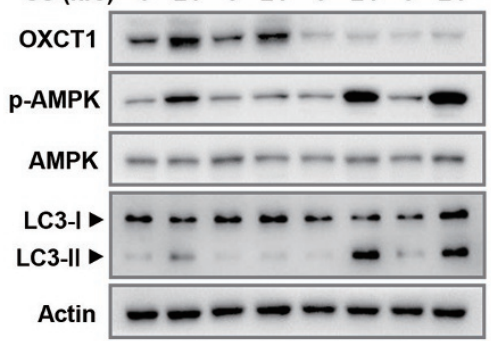

H

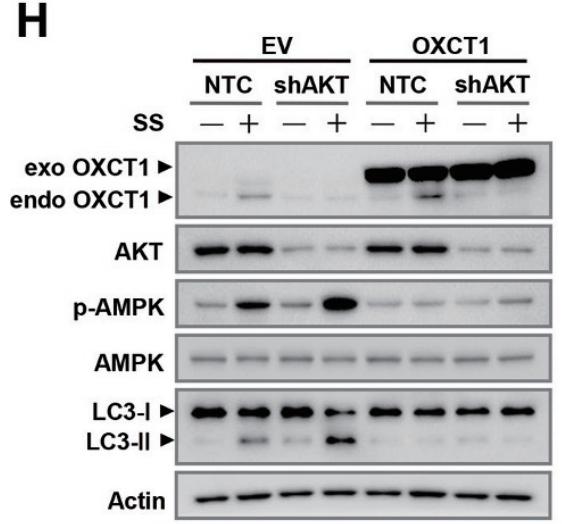

D

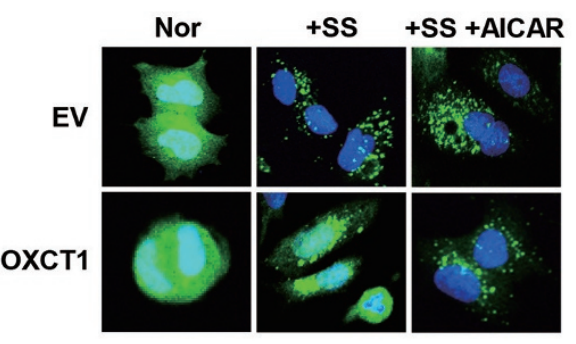

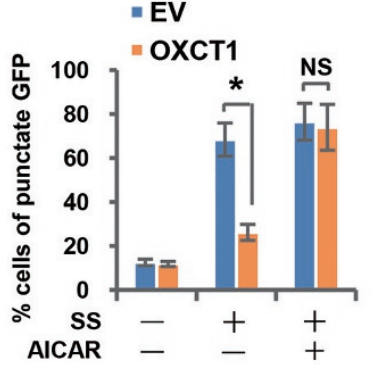

G
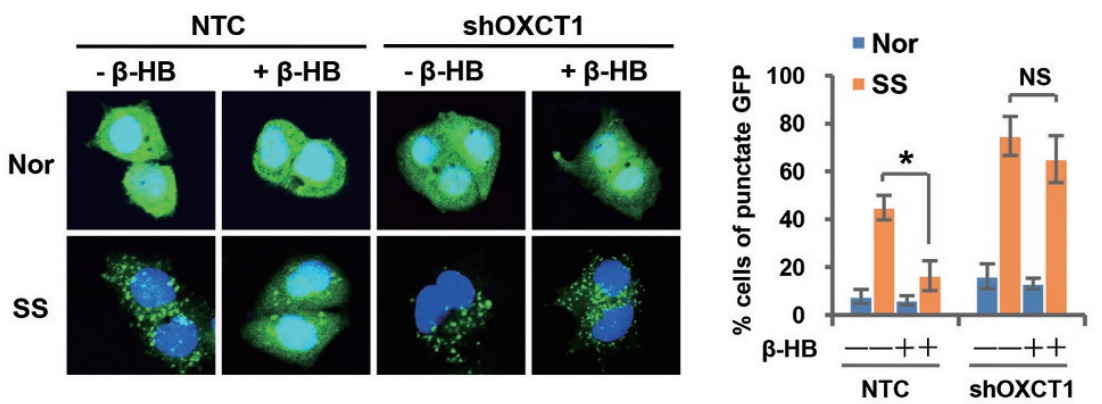

I
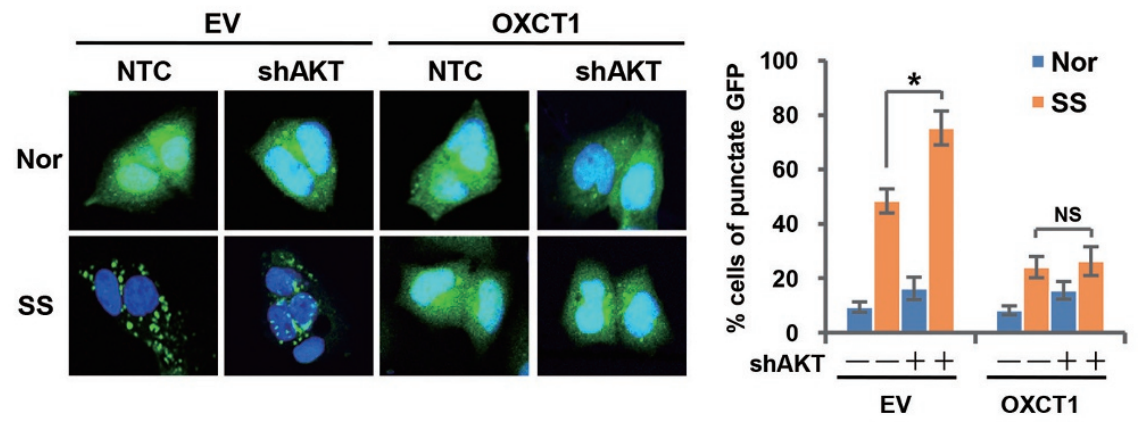
Figure 4 Ketolysis suppresses AMPK activation and autophagy in nutrition-starved HCC cells by elevating ATP production. (A) Western blot analysis of OXCT1 and LC3-I/II levels in HepG2 cells stably expressing OXCT1 shRNA or NTC under normal or SS conditions for $24 \mathrm{~h}$. (B) HepG2 cells stably expressing OXCT1 shRNA or NTC were further infected with viruses expressing pSIN-GFP-LC3 and cultured under Nor or SS conditions for $24 \mathrm{~h}$. The percentage of cells exhibiting punctate fluorescence under fluorescence microscopy was calculated relative to all GFP-positive cells. Data were presented as mean \pm SD. ${ }^{*} P<0.05$ as compared with Nor-treated NTC group; ${ }^{*} P<0.05$ as compared with SS-treated NTC group. (C) HepG2 cells stably expressing OXCT1 shRNA (targeting 3'UTR) were further infected with viruses expressing pSIN-3×flag-OXCT1 or pSIN-EV. Each group of cells was treated with vehicle (DMSO) or AICAR (1 mM) for $2 \mathrm{~h}$ prior to serum starvation for 24 h. OXCT1 and LC3-I/II protein levels were detected by western blot. (D) HepG2 cells in C were further infected with viruses expressing pSIN-GFP-LC3. Each group of cells was treated with vehicle (DMSO) or AICAR (1 mM) for $2 \mathrm{~h}$ prior to serum starvation for $24 \mathrm{~h}$. Percentage of cells exhibiting punctate fluorescence under fluorescence microscopy was calculated relative to all GFP-positive cells. Data were presented as mean \pm SD. ${ }^{*} P<0.05$ as compared between the indicated group. (E) HepG2 cells stably expressing OXCT1 shRNA (targeting 3'UTR) or NTC were further infected with viruses expressing pSIN$3 \times$ flag-OXCT1 or pSIN-EV. Each group of cells was cultured under normal $(0 \mathrm{~h})$ or serum starvation (SS) conditions for 12 or $24 \mathrm{~h}$, followed by western blot analyzing OXCT1, AMPK and p-AMPK levels. (F) HepG2 cells stably expressing OXCT1 shRNA or NTC were cultured under normal $(0 \mathrm{~h})$ or SS conditions for $24 \mathrm{~h}$ in the presence or absence of $5 \mathrm{mM} \beta-\mathrm{HB}$, followed by western blot analysis of OXCT1, AMPK, p-AMPK and LC3-I/II. (G) HepG2 cells in F were further infected with viruses expressing pSIN-GFP-LC3. Cells was cultured under Nor or SS conditions for $24 \mathrm{~h}$ in the presence or absence of $5 \mathrm{mM} \beta$-HB. Percentage of cells exhibiting punctate fluorescence under fluorescence microscopy was calculated relative to all GFP-positive cells. Data were presented as mean \pm SD. ${ }^{*} P<0.05$ as compared between the indicated group. (H) HepG2 cells stably expressing pSIN-3×flag-OXCT1 or EV were further infected with viruses expressing AKT shRNA or NTC. Cells were cultured under normal or SS conditions for $24 \mathrm{~h}$, followed by western blot analyzing AKT, OXCT1, AMPK, p-AMPK and LC3-I/II levels. (I) HepG2 cells in $\mathbf{H}$ were further infected with viruses expressing GFP-LC3. Each group of cells was cultured under Nor or SS conditions for $24 \mathrm{~h}$. Percentage of cells exhibiting punctate fluorescence under fluorescence microscopy was calculated relative to all GFP-positive cells. Data were presented as mean \pm SD. ${ }^{*} P<0.05$ as compared between the indicated group.

binds to these GC-box motifs in HepG2 cells, which was gradually enhanced by serum withdrawal (Figure $3 \mathrm{~J}$ ), indicating that OXCT1 is a direct transcriptional target of SP1. Taken together, these data demonstrate that SP1 binds to OXCT1 promoter and activates OXCT1 expression in serum-starved cells.

AKT, as a kinase, is known to phosphorylate SP1, leading to SP1 activation [30]. Our western blot analysis showed that phosphorylation of threonine 453 on SP1 was markedly enhanced by serum depletion, which was blocked by AKT inhibition with LY-294002 in HepG2 cells (Supplementary information, Figure S3E and S3F), suggesting that AKT activated SP1 under serum starvation. Moreover, forced expression of constitutively active myristoylated AKT (myr-Akt) resulted in enhanced SP1 phosphorylation and OXCT1 protein expression in HepG2 cells (Figure 3K). Importantly, knockdown of SP1 with shRNAs abolished the induction of OXCT1 expression by myr-Akt (Figure 3K), providing evidence that SP1 acts downstream of AKT in the regulation of OXCT1. Taken together, these data demonstrate that the AKT pathway, activated by mTORC2 under serum starvation conditions, transcriptionally stimulates OXCT1 expression via phosphorylating SP1 (Figure 3L). Further, metabolic analysis by GC-MS revealed that knockdown of AKT or SP1 abolished serum starvation-induced catabolism of $\left[2,4_{-}{ }^{13} \mathrm{C}_{2}\right] \beta$-HB into metabolites of TCA cycle in HepG2 cells (Figure 3M), confirming that AKT and SP1 are critical for ketolysis activation in serum-deprived HCC cells. Of note, we did not observe the phosphorylation of AKT at serine 473 and phosphorylation of SP1 at threonine 453 in HepG2 cells under glucose or glutamine starvation conditions (Supplementary information, Figure $\mathrm{S} 3 \mathrm{G}$ ), which is consistent with our findings that induction of OXCT1 expression was limited to the stress of serum starvation in HCC cells (Figure 1A and 1B).

Ketolysis suppresses AMPK activation and autophagy in nutrition-starved HCC cells by elevating ATP production

Autophagy is activated as a cellular protective mechanism to sustain cell survival during serum starvation [31, 32]. However, prolonged or excessive autophagy could ultimately cause non-apoptotic type II programmed cell death $[33,34]$. To explore whether OXCT1 is involved in autophagy regulation under serum deprivation, we analyzed the accumulation of LC3-II protein, a hallmark of autophagy. Western blot results showed that serum starvation-induced accumulation of LC3-II was further enhanced by OXCT1 knockdown in HepG2 cells (Figure 4A). Autophagy was also analyzed by transfecting HepG2 cells with the GFP-LC3 fusion protein (Figure 4B). Consistent with western blot results, OXCT1 knockdown increased the percentage of cells displaying punc- 
A

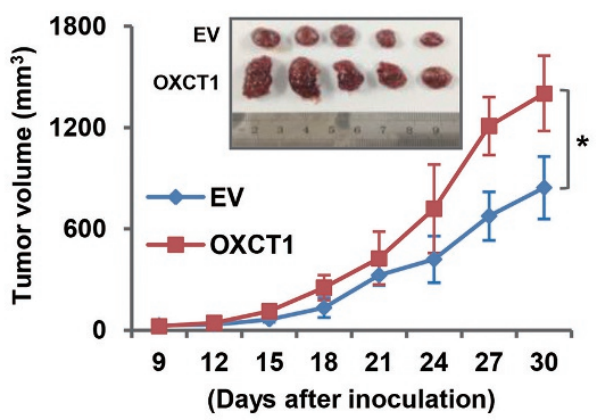

C

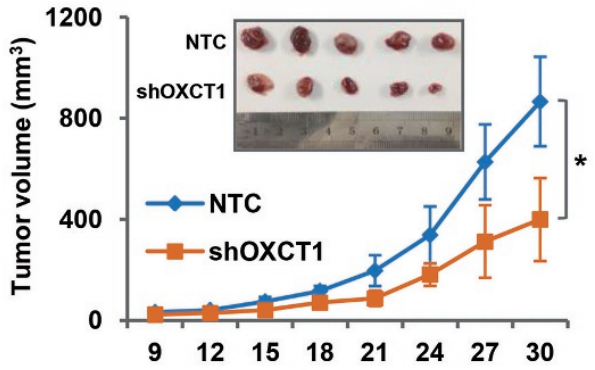

(Days after inoculation)

E

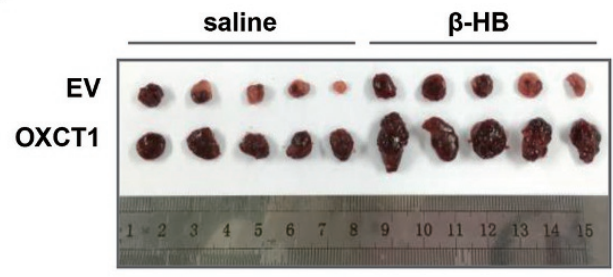

$\mathbf{F}$

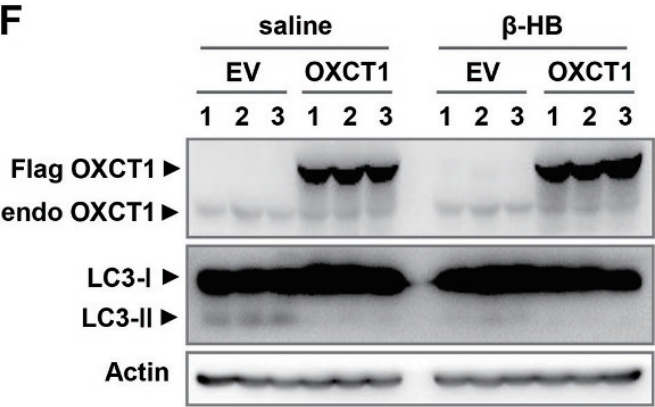

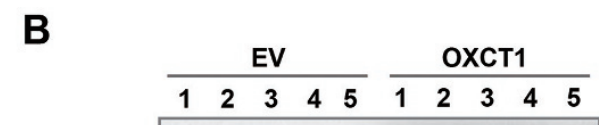

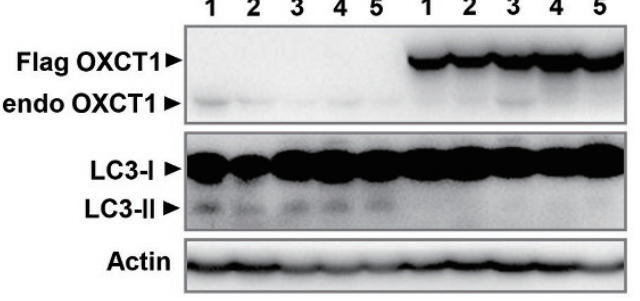

D
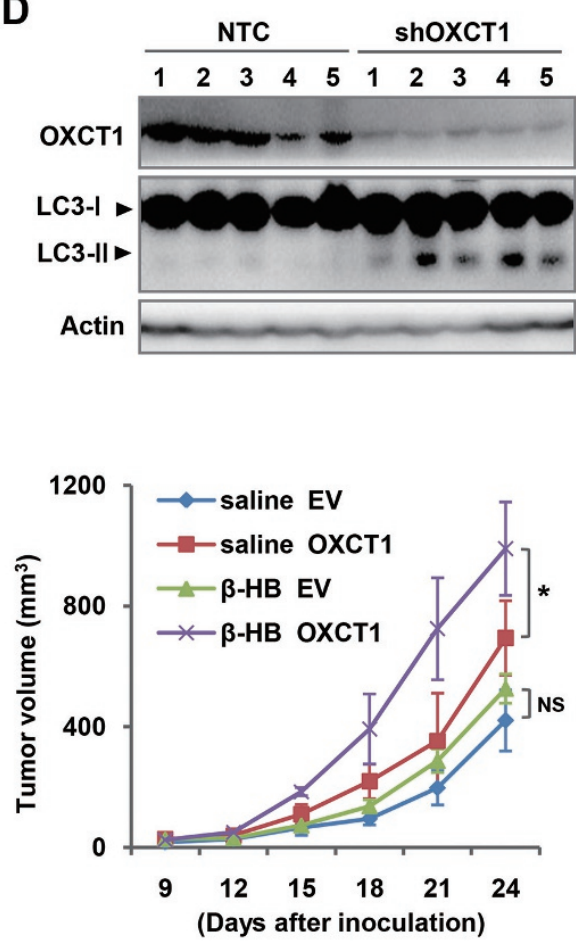

Figure 5 OXCT1 promotes HCC progression in vivo. (A) HepG2 cells stably expressing OXCT1 or EV were injected subcutaneously into nude mice ( $n=5$ for each group). Tumor growth was measured starting from 9 days after inoculation, and tumors were extracted and compared at the end of the experiment. Data shown are mean \pm SD. ${ }^{*} P<0.05$ as compared with EV group. (B) Protein levels of OXCT1 and LC3-I/II were determined by western blot using the lysates from five independent tumors of each group as in A. (C) HepG2 cells stably expressing OXCT1 shRNA or NTC were injected subcutaneously into nude mice ( $n=5$ for each group). Tumor growth was measured starting from 9 days after inoculation, and tumors were extracted and compared at the end of the experiment. Data shown are mean \pm SD. ${ }^{*} P<0.05$ as compared with NTC group. (D) Protein levels of OXCT1 and LC3-I/II were determined by western blot using the lysates from five independent tumors of each group as in C. (E) HepG2 cells stably expressing OXCT1 or EV were subcutaneously injected into nude mice $(n=5$ for each group). Starting from the inoculation, mice were administered with either saline or saline containing $\beta-\mathrm{HB}(500 \mathrm{mg} / \mathrm{kg})$ by daily i.p. injection. Tumor growth was measured starting from 9 days after inoculation. Tumors were extracted and compared at the end of the experiment. Data shown are mean \pm SD. ${ }^{*} P<0.05$ as compared between the indicated groups. (F) Protein levels of OXCT1 and LC3-I/II were determined by western blot using the lysates of tumor tissues from each group as in $\mathbf{E}$. 
A

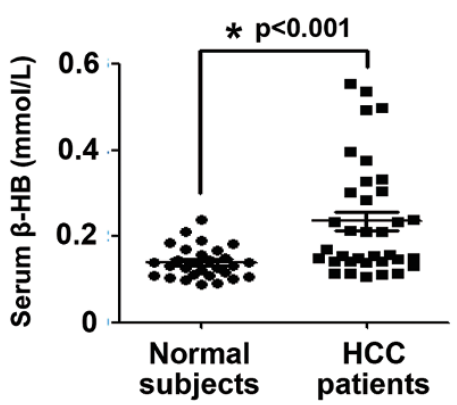

B

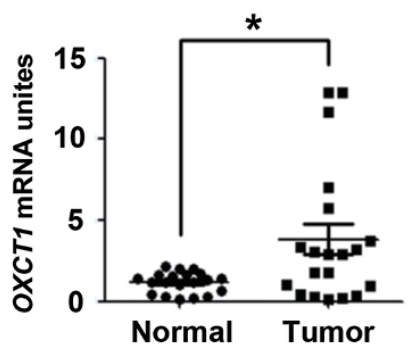

C

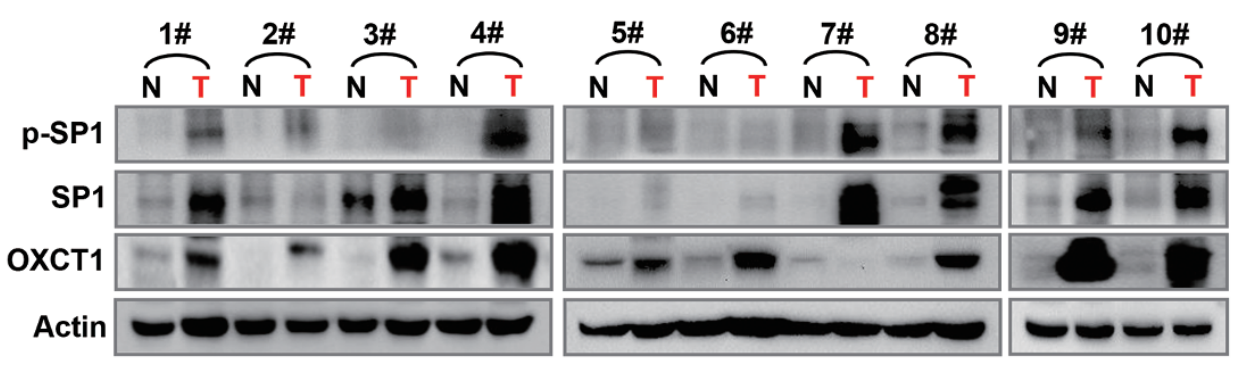

D

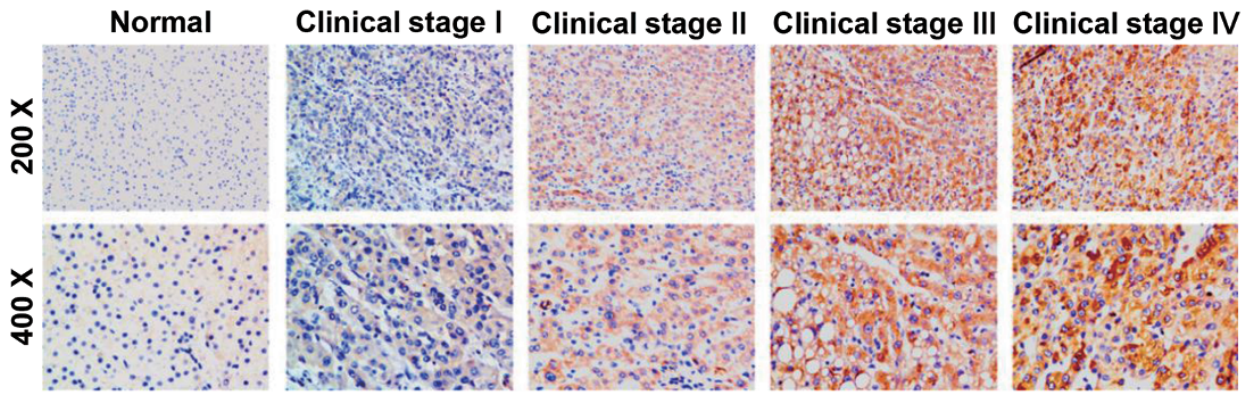

E

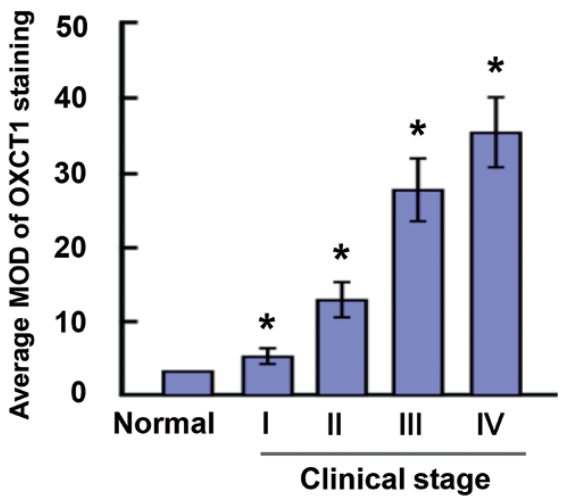

F

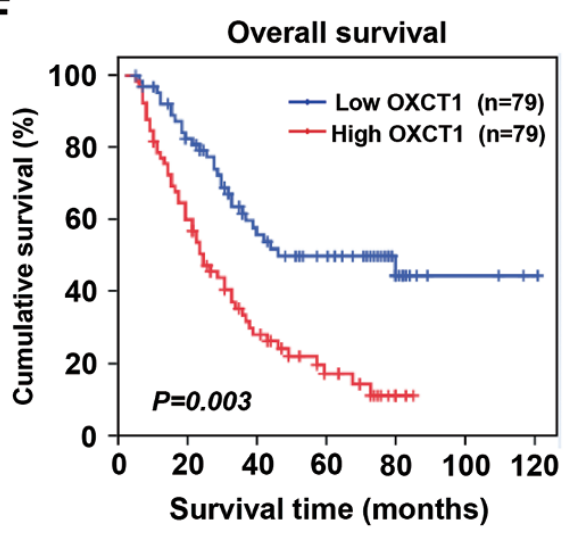

Figure 6 Aberrant OXCT1 expression predicts patient mortality in clinical HCC. (A) Serum $\beta$-HB levels were measured in 29 normal subjects $(n=29)$ and 35 HCC patients $(n=35)$. Data were presented as mean \pm SD. ${ }^{*} P<0.001$ as compared between two groups. (B) mRNA levels of OXCT1 were determined by qRT-PCR in 20 pairs of clinically matched tumor adjacent non-cancerous liver tissues (normal) and human HCC tissues (tumor). Data were presented as mean $\pm \mathrm{SD}(n=20)$. ${ }^{\star} P<$ 0.05 as compared between two groups. (C) p-SP1, SP1 and OXCT1 levels were determined by western blot using the paired tumor adjacent non-cancerous liver tissues (normal) and human HCC tissues (tumor). (D) Representative IHC analysis of OXCT1 expression in normal liver tissues (normal) and HCC specimens of different clinical stages (I-IV) was shown. (E) Statistical quantification of the mean optical density (MOD) values of OXCT1 staining in IHC assay between normal liver tissues and HCC specimens of different clinical stages (I-IV). The MOD of OXCT1 staining increases as HCC progresses to a higher clinical stage. Data were presented as mean \pm SD. ${ }^{*} P<0.05$ as compared with normal control group. (F) Kaplan-Meier curves with univariate analyses for patients with low versus high OXCT1 expression; $n=79$ for each group. 
tate fluorescence under serum starvation (Figure 4B), confirming that knockdown of OXCT1 promotes cell autophagy under stressed conditions. On the other hand, restoring OXCT1 expression in OXCT1-knockdown HepG2 cells prevented cells from serum deprivation-induced autophagy, as assessed by LC3-II protein level as well as punctate GFP-LC3 fluorescence (Figure 4C and 4D), highlighting the protective effect of OXCT1 by restricting autophagy under serum deprivation. However, AICAR, an AMPK activator, abolished the protective effect of OXCT1 overexpression in HepG2 cells (Figure 4C and 4D), suggesting that AMPK pathway might be involved in OXCT1-regulated autophagy under serum starvation.

AMPK is a key sensor of energy status and is activated in response to increased cellular AMP/ATP ratios after LKB1-mediated phosphorylation of the conserved residue Thr172 [35, 36]. Activation of AMPK has been reported to facilitate cell autophagy [37]. Consistent with previous reports, we observed increased phosphorylation of AMPK, indicating its activation during serum starvation (Figure 3A). To investigate whether AMPK is involved in the autophagy regulated by OXCT1 under serum starvation, we performed western blot to examine the Thr172 phosphorylation of AMPK in HepG2 cells. We found that forced expression of OXCT1 attenuated serum starvation-induced AMPK phosphorylation, while knockdown of OXCT1 elevated AMPK phosphorylation under serum starvation, which was abolished by overexpression of OXCT1 back into the HepG2 cells (Figure $4 \mathrm{E})$. Taken together, these data suggest that AMPK pathway is involved in OXCT1-regulated autophagy under nutrition-deficient conditions in HCC cells. Since our data showed that OXCT1 was critical for maintaining cellular ATP levels by catalyzing ketone bodies under nutrition stressed conditions (Figure $2 \mathrm{~F}$ and $2 \mathrm{H}$ ), we next studied the effect of supplement of exogenous $\beta$-HB on AMPK activation as well as cell autophagy in stress conditions. Western blot analysis revealed that exogenous $\beta$-HB attenuated serum starvation-induced AMPK phosphorylation in control cells but not in HepG2 cells expressing OXCT1 shRNAs (Figure 4F). Consistently, LC3-II protein as well as LC3 punctate fluorescence analysis revealed that supplement of exogenous $\beta$-HB protected cells from nutrition deprivation-induced autophagy in control but not in OXCT1-knockdown HepG2 cells (Figure 4F and 4G).

Further analysis showed that knockdown of AKT by shRNA boosted serum starvation-induced AMPK phosphorylation, LC3-II protein accumulation, and GFPLC3 puncta formation, which were abolished by forced expression of OXCT1 in HepG2 cells (Figure 4H and

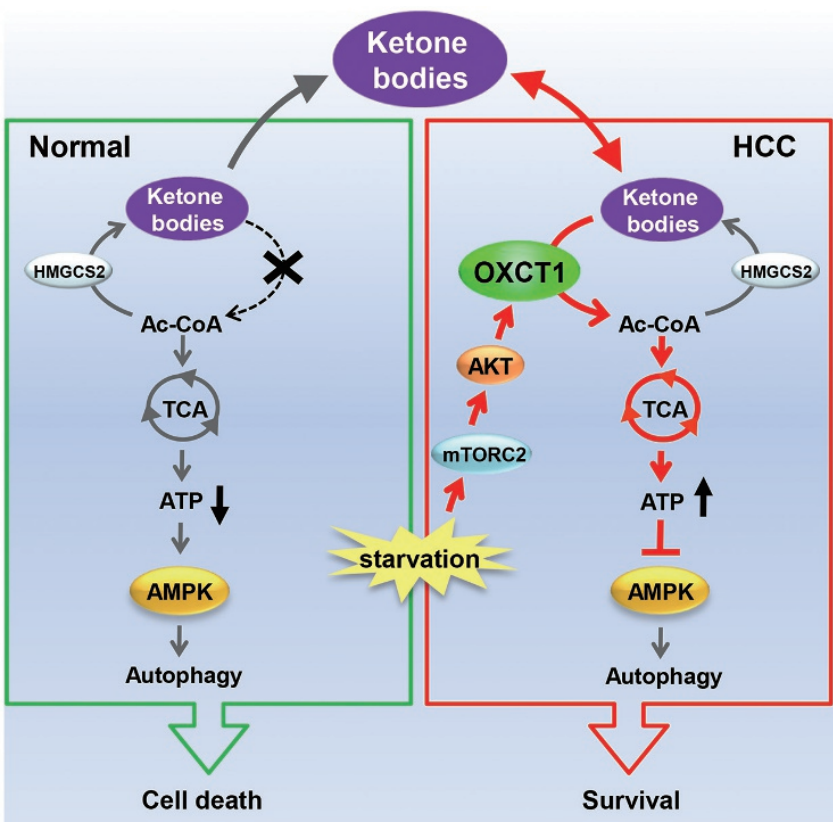

Figure 7 Ketolysis induced by nutrition deprivation suppresses autophagy to promote liver cancer progression. Schematic showing that, unlike normal liver cells, HCC could use ketone bodies produced by cancer cell themselves, normal liver cells or other potential sources from the tumor microenvironment. Mechanistically, serum starvation-induced OXCT1 expression via mTORC2-AKT-SP1 signaling promotes ketone body catabolism to elevate cellular ATP levels in HCC cells, which inhibits AMPK activation and protects liver cancer cells from excessive autophagy under nutrition limitation conditions.

4I). These data were consistent with our observation that AKT acts upstream of OXCT1, through regulating its transcription by activating SP1 under serum starvation (Figure 3L). Collectively, our data show that serum starvation stimulates AKT activation and its downstream OXCT1 expression, which facilitates ketone body catabolism to sustain cellular energy homeostasis. As a consequence, AMPK activity was inhibited to prevent excessive autophagy in nutrition-deficient HCC cells.

\section{OXCT1 promotes HCC progression in vivo}

The microenvironment of solid tumors provides a complicated milieu for tumor cell survival and growth, which features hypoxia, low $\mathrm{PH}$, nutrient stress, among others. To evaluate the effect of OXCT1 on cancer cell proliferation in vivo, xenograft experiments in nude mice were performed. Forced expression of OXCT1 in HepG2 cells significantly accelerated its xenograft tumor growth (Figure 5A and Supplementary information, Figure S4A). Western blot revealed that tumors generated from OXCT1-overexpressing HepG2 cells had lower LC3-II 
levels than control group (Figure 5B), suggesting less autophagy in OXCT1-overexpressing xenograft tumor tissues, which is consistent with the data obtained from cells in culture (Figure 4C and 4D). On the other hand, stable knockdown of OXCT1 using shRNAs markedly retarded the growth of HepG2 cell xenografts (Figure 5C and Supplementary information, Figure S4B). Increased LC3-II levels were observed in tumor tissues generated from OXCT1-knockdown HepG2 cells (Figure 5D), further highlighting the ability of OXCT1 to restrict autophagy, by which it promotes cancer cells to adapt to tumor microenvironment.

High-fat low-carbohydrate ketogenic diets have been studied in various tumor models for their effect on tumorigenesis, including lung cancer and medulloblastoma $[38,39]$. However, it has never been documented whether high levels of ketone bodies have an effect on liver cancer progression in vivo. Encouraged by our results that ketolysis activation via OXCT1 promotes HCC proliferation both in vitro and in vivo, we tested the effect of ketogenic diet on HCC growth in mouse models. Surprisingly, ketogenic diet did not promote HCC growth regardless of OXCT1 overexpression (Supplementary information, Figure S4C). Analysis of the glucose and ketone bodies in mice indicated that, while the ketogenic diet we used in mice resulted in higher serum levels of ketone bodies, it also substantially decreased serum glucose levels and mouse body weights (Supplementary information, Figure S4D), which potentially diminished the glucose supply that fuels tumor progression via the Warburg effect. Hence, we next modified our strategy of ketone body administration by daily intraperitoneal (i.p.) injection of $\beta$-HB. Our analysis confirmed elevated serum levels of ketone bodies but no fluctuation in glucose levels and mouse body weights after i.p. injection of $\beta$-HB (Supplementary information, Figure S4E). Intriguingly, we observed that the ketone body administration significantly promoted $\mathrm{HCC}$ cell growth in vivo when OXCT1 was overexpressed (Figure 5E). Western blot analysis further revealed that, similar to OXCT1 overexpression, $\beta$-HB administration also reduced LC3-II levels in tumor tissues (Figure 5F), confirming the protective effect of $\beta$-HB administration on cancer cell survival in vivo.

\section{Aberrant OXCT1 expression predicts patient mortality in clinical HCC}

We then explored the potential clinical significance of ketolysis re-activation as a result of induced expression of OXCT1 in HCC development. First, we sought to determine the levels of serum $\beta$-HB and OXCT1 expression in the patients with liver cancer. Our results revealed that
HCC patients have significantly higher levels of serum $\beta$-HB as compared with normal subjects (Figure 6A). Ketolysis-related enzymes were also studied in 20 paired HCC lesions and adjacent noncancerous tissue samples. Among the enzymes, only OXCT1 mRNA levels were significantly increased in HCC lesions compared with adjacent normal tissues (Figure 6B and Supplementary information, Figure S5A). Although $\mathrm{BDH} 1$ expression is increased upon serum starvation in HCC cell lines, we did not observe significant difference in its mRNA levels between HCC lesions and adjacent normal tissues (Supplementary information, Figure S5A). Furthermore, western blot analysis showed that, besides the increase in total and phosphorylated SP1 levels as previously reported [30], OXCT1 protein levels were increased in $\mathrm{HCC}$ tissue compared with adjacent normal tissue (Figure $6 \mathrm{C})$.

Moreover, immunohistochemistry (IHC) was employed to analyze OXCT1 expression in a retrospective cohort of 158 clinicopathologically characterized HCC cases, including 16 cases of stage I $(10.1 \%), 88$ cases of stage II $(55.7 \%), 43$ cases of stage III $(27.2 \%)$, and 11 cases of stage IV (7\%) liver cancer, based on the TNM staging (Supplementary information, Table S1). IHC results revealed that OXCT1 protein was generally negative in normal liver, weak in early-stage HCC (TNM stages I and II) and abundant in late-stage HCC (TNM stages III and IV) (Figure 6D). High power microscopy (400× magnification) revealed enrichment of OXCT1 expression in malignant hepatocytes. Quantitative analysis of the IHC images revealed that OXCT1 expression level in clinical stages I-IV primary tumors was significantly increased compared with that in normal liver tissue (Figure 6E). Moreover, expression of OXCT1 was drastically upregulated in late-stage HCC (stages III and IV) as compared with early-stage HCC (stages I and II; Figure 6E and Supplementary information, Table S2), suggesting that OXCT1 expression is gradually elevated as HCC progresses to a higher clinical stage. Further, Spearman analysis showed correlations between OXCT1 expression and patient clinicopathological characteristics, including survival time, vital status, clinical stage, and tumor size (Supplementary information, Table S3), further indicating a strong association of OXCT1 expression with HCC clinical staging and patient survival. Univariate analysis showed that, together with TNM stage and tumor size, OXCT1 level is a significant prognostic factor and is predictive of the overall survival of HCC patients (Supplementary information, Table S4). Finally, Kaplan-Meier tests indicated that OXCT1 expression in HCC patients was significantly associated with survival time, with patients expressing low levels of OXCT1 in 
their HCC lesions surviving much longer than those with high OXCT1 expression (Figure 6F), suggesting that OXCT1 protein may represent a promising prognostic biomarker in HCC.

\section{Discussion}

Since nutrient-limiting conditions are not uncommon during cancer development [40], we are interested in what pathways or which fuels might provide alternative sources for energy production in starved cancer cells. Using a screening approach to determine changes of lipid metabolism-related gene expression induced by nutrition stresses, we have discovered that the expression level of OXCT1, a key enzyme for ketone body catabolism, is dramatically increased in serum-starved liver cancer cells (Figure 1A and 1B). Further GC-MS analysis reveals that serum starvation activates $\beta$-HB catabolism for energy production and cancer progression in liver cancer cells, but not in normal liver cells or cancer cells cultured under normal conditions (Figure 1D and $1 \mathrm{~F}$ ). $\beta$-HB is the most abundant circulating ketone body in the organisms, and is a "super-fuel" that burns more efficiently to produce ATP energy than glucose or fatty acid during fasting [41]. However, previous studies showed that OXCT1 is expressed in the mouse embryonic liver, and then its expression gradually decreased during hepatocyte maturation [19]. Thus, despite being the main organ for ketogenesis, the liver expresses little OXCT1 relative to other organs. It is therefore generally believed that adult liver cells do not use ketone bodies for energy due to lack of OXCT1 expression. It is very intriguing to uncover in the present study that expression of OXCT1 is activated by serum starvation-triggered mTORC2AKT-SP1 signaling in HCC cells (Figure 3), resulting in utilization of ketone bodies for ATP production and cell proliferation via repression of AMPK activation and autophagy (Figure 4). This finding indicates that liver cancer cells consume ketone bodies as a convenient fuel to promote survival and progression under stressed conditions. In short, we uncover here a rewired novel metabolic pathway by which ketolysis is re-activated in nutrition-limiting HCC cells for energy supply and cancer progression.

As the sole mechanism to degrade large structures such as organelles and protein aggregates, autophagy is an evolutionarily conserved process in eukaryotic cells that eat parts of themselves in order to survive. The autophagy level is very low under homeostasis conditions. However, when cells encounter environmental stresses such as nutrition or oxygen deprivations, autophagy increases dramatically as a mechanism of adaptation for overall survival $[42,43]$. While increasing evidence links autophagy to cancer, the roles of autophagy in cancer development still remain controversial. Early reports established that cancer cells often have lower autophagic capability or protein degradation rates than their normal counterparts [44]. Autophagy-deficient transgenic mice often caused a high incidence of lung cancer, hepatocellular carcinoma and lymphoma, which suggests that dysregulation of autophagy contributes to tumorigenesis [45, 46]. However, significant autophagy did occur in amino acid-, glucose-, or oxygen-deprived cancer cells, which might be triggered to protect tumor cells by degrading the damaged organelles [33, 47, 48]. Signaling from energy- or nutrient-sensitive AMPK is essential for the nutrient sensing of the autophagy pathway, underlining energy limitation as a major cause for autophagy. Hence, we envision that, if activation of AMPK is to sense the low energy stress to trigger degradation of cellular components, as autophagy is so defined, for the overall survival of cancer cell population, a better strategy for cancer cells to adapt to nutritional stresses would be to exploit additional energy sources to bypass autophagy. Indeed, we observed that AMPK-mediated autophagy was drastically attenuated by OXCT1 induction in serum-starved HCC cells. Mechanistically, induction of OXCT1 promotes ketolysis, leading to elevated ATP levels and suppressed AMPK activity. Thus, here we provide a new mechanism whereby cancer cells rewire metabolic pathway for extra energy sources to bypass excessive autophagy during cancer development.

We observed that ketogenesis was critical for OXCT1-stimulated HCC cell survival and proliferation under stress conditions (Figure 2). When HMGCS2, a rate-limiting ketogenesis enzyme, was knocked down in the absence of ketone bodies in the culture medium, the growth advantage conferred by OXCT1 expression was abolished. Addition of ketone bodies to the culture medium restored OXCT1-stimulated cell growth (Supplementary information, Figure S2I). These results demonstrate that OXCT1-mediated HCC survival and growth depend on consumption of ketone bodies. Of note, HCC cells lacking both HMGCS2 and OXCT1 exhibited a lower proliferation rate compared with cells harboring both (Supplementary information, Figure S2I). This result suggests that an active flow of ketogenesis and ketolysis is important for an optimal proliferation of HCC cells, the mechanism of which warrants future investigation. It is possible that besides modulating ATP levels, an active ketogenesis and ketolysis flow may have broader effects on HCC development. For instance, previous reports documented signaling roles of ketone bodies via HDAC inhibition [49] or GPR109A signaling [50]. More- 
over, consistent with the ability of OXCT1 to catalyze the conversion of succinyl-CoA to succinate, OXCT1 overexpression led to elevation of both ${ }^{13} \mathrm{C}$-incorporated succinate and ${ }^{12} \mathrm{C}$-succinate levels (Supplementary information, Figure S1F). The increased ${ }^{12} \mathrm{C}$-succinate potentially represents succinyl-CoA-converted succinate during $\beta$-HB catabolism (Supplementary information, Figure S1G). Thus, further study is warranted to explore the possibility that OXCT1-stimulated increase in succinate level and subsequent succinate metabolism may play additional pro-tumor roles.

It is intriguing that we identified mTORC2-AKT as the upstream factor to trigger OXCT1 expression under serum starvation condition. AKT is a well-known survival factor whose activation contributes to human HCC development [51]. It is worthwhile to note that, while AKT was reported to be inactivated by growth factor deprivation under serum starvation [52], serum withdrawal also widely increased AKT phosphorylation and activity in human cancer cells under different conditions [25, 27, 53], especially in serum-starved hepatoma cells [26]. Those results are in agreement with the pro-survival role of AKT. While we could not rule out other mechanisms that might be involved in sustaining OXCT1 expression, it is important to note that we have provided several lines of evidence to clearly establish the regulatory role of mTORC2-AKT-SP1-OXCT1 in the re-induction of hepatocellular ketolysis in cancer cells (Figure 3C-3G, 3K and $3 \mathrm{M})$. One of the well-defined mechanisms for AKT to promote survival is to counteract AMPK activation by mTORC2-AKT-SP1 pathway to suppress autophagy [54]. Hahn-Windgassen and colleagues first provided the evidence that AKT diminished AMPK activity by increasing cellular ATP levels [55]. Here, we provide new evidence that mTOR-AKT-mediated induction of OXCT1 was sufficient to elevate cellular ATP production by stimulating ketone body catabolism, which subsequently diminished AMPK activity and inhibited AMPK-mediated autophagy in nutrition-limited liver cancer cells (Figure 7). Our novel results here added another layer of complexity to the multifaceted roles of AKT in promoting cancer cell survival and proliferation.

The cell growth assays in vitro and in vivo suggest that OXCT1 expression in HCC cells promotes cancer progression (Figures 2G, 2I, 5A and 5C). This is very interesting considering that $\mathrm{OXCT} 1$ expression and ketolysis are absent in normal hepatocellular cells, positioning OXCT1 as a potential target for HCC therapy. Indeed, our xenograft experiments demonstrated that elevated ketone body levels significantly promoted HCC cell growth in vivo when OXCT1 was overexpressed (Figure $5 \mathrm{E})$. These results prompted us to explore directly the potential clinical relevance of the deregulated ketone body metabolism to HCC development. Significantly, we observed elevated serum $\beta$-HB levels in HCC patients compared with normal controls (Figure 6A), suggesting that ketone bodies, produced by both malignant and normal tissues, are readily available as a potential fuel source for cancer development. We further observed clinical correlation of aberrant OXCT1 expression with HCC development and patient survival, suggesting that deregulated expression of OXCT1 predicts patient mortality (Figure 6D-6F). This correlation might be highly relevant for understanding the pathogenic role of the deregulated ketone body metabolism during liver cancer development, further suggesting a potential rationale to target HCC-specific OXCT1 pathway in general for liver cancer therapy.

\section{Materials and Methods}

\section{Cell culture and reagents}

Human HepG2, Hep3B, PLC, HEK293 and HEK293T cells were cultured in DMEM medium containing $25 \mathrm{mM}$ glucose, 4 $\mathrm{mM} \mathrm{L}$-glutamine and $1 \mathrm{mM}$ pyruvate (Gibco, 12800). THLE3 cells were cultured in BEGM (LONZA, CC3170). The media were supplemented with $1 \%$ penicillin-streptomycin and $10 \%$ FBS. For serum starvation, cells were washed twice with PBS and cultured in the medium without FBS. DL- $\beta$-hydroxybutyrate (H6501), LY294002 (L9908), PD98059 (P215), EVP4593 (SML0579) and AICAR (A9978) were purchased from Sigma-Aldrich. Compound C (Santa cruz, sc-200689), Z-VAD-FMK (Selleck, S7023), Rapamycin (Selleck, S1039), Mithramycin A (TOCRIS, 1489) were also used to treat cells with vehicle as control.

\section{$q R T-P C R$}

Total RNA was isolated using Trizol followed by DNase (Ambion) treatment and reverse transcription with iScript cDNA Synthesis Kit (Bio-Rad). qRT-PCR was performed using SYBR Green master mix (Vazyme) on a Bio-Rad iCycler. Primer sequences used were shown in Supplementary information, Table S5. All samples were normalized to $18 \mathrm{~S}$ rRNA.

\section{Western blot}

Cells were lysed with RIPA buffer and equal amounts of protein in the lysates were boiled and fractionated by $7 \%-10 \%$ SDSPAGE. Primary antibodies against the following proteins were used: BDH1, OXCT1, OXCT2, ACAT1, ACAT2, SP1, HMGCS2, HMGCL, and $\beta$-actin (from Proteintech); IкB $\alpha$ and NF-B (from Santa Cruz); AMPK, p-AMPK (Thr172), AKT, p-AKT (ser473), ERK1/2, p-ERK1/2 (Thr202/Tyr204) (from Cell Signaling Technology); p-SP1 (Thr453), HMGCS2 (from Abgent); LC3 (Novus Biologicals). Signal was detected using Western ECL Substrate (Bio-Rad).

\section{GC-MS analysis of metabolites}

Cells were incubated in the culture medium supplemented with $5 \mathrm{mM}\left[2,4-{ }^{13} \mathrm{C}_{2}\right]$-labeled $\beta$-hydroxybutyrate (Sigma Aldrich, $674117)$ under the indicated conditions. Metabolites were extract- 
ed from cells as previously described [56, 57]. Briefly, each group of cells was collected and immediately flash-frozen in liquid $\mathrm{N}_{2}$, metabolites were extracted with ice-cold methanol and lysates were centrifuged at $18000 \times g$ for $15 \mathrm{~min}$ to remove protein. The supernatant was dried in an evaporator and resuspended in 200 $\mu \mathrm{l}$ of pyridine. Metabolites were further derivatized by addition of $50 \mu \mathrm{l}$ of MTBSTFA containing $1 \% \mathrm{t}$-BDMCS at $60^{\circ} \mathrm{C}$ for $1 \mathrm{~h}$. Samples were analyzed using Agilent 5MS column in the Agilent 7890/5975C GC/MS system (Agilent Technologies, Santa). Peaks representing each metabolite were extracted and integrated using MassHunter software (Agilent Technologies). ${ }^{13} \mathrm{C}$-Labeled metabolite data are presented as percentage of ${ }^{13} \mathrm{C}$-labeled metabolites, which was calculated by dividing the labeled ions with total ion intensity. The labels are irrespective of carbon position. The distribution of mass isotopologues was corrected for natural abundance. We used IsoCor, a scientific software designed for the purpose of isotope labeling experiments, to correct the raw MS data for both all naturally-occurring isotopes and purity of the isotopic tracer [58]. The website for the software (IsoCor) is http://metasys.insa-toulouse.fr/software/isocor/.

\section{Cellular ATP measurement}

ATP levels were determined by luciferin-luciferase-based ATP Assay Kit (Promega) with luminescence reader (Promega). Luminescence was normalized to the protein concentration.

\section{Plasmids and establishing stable cells}

shRNAs in PLKO vector against OXCT1, BDH1, AKT, SP1 and Rictor were commercially purchased (Sigma-Aldrich). Coding sequences of human $O X C T 1, B D H 1, S P 1$ or GFP-LC3 was subcloned into pSIN lentiviral vector. $O X C T 1$ was also inserted into pSIN-3 $\times$ flag lentiviral vector. pBabe-puro-myr-flag-AKT vector was purchased from Addgene (Plasmid \#15294). Oligonucleotide sequences of shRNA targeting OXCT1 3'UTR (2 674-2 696) was inserted into PLKO vector at EcoRI and AgeI sites (5'-CCGGAGGGCTGTGGGATAATTTACCCTCGAGGGTAAATTATCCCACAGCCCTTTTTTG-3', 5'-AATTCAAAAAAGGGCTGTGGGATAATTTACCCTCGAGGGTAAATTATCCCACAGCCCT-3'). Transduction and viral infection was performed as previously described [59].

\section{$\beta-H B$ and AcAc measurements}

$\beta$-HB Assay Kit (Cayman) was used to measure extracellular $\beta$-HB in cell culture medium as well as intracellular $\beta$-HB in cell lysates. Intracellular AcAc was measured using AcAc Assay Kit (BioVision). All measurements followed the manufacturer's instructions and the values were normalized to protein concentration.

\section{Dual luciferase reporter assay}

Human OXCT1 promoter sequences were inserted into the pGL2 basic luciferase reporter vector (Promega), designated as pGL2-OXCT1 promoter. Two GC-box and their mutated sequences were inserted into the pGL2-promoter reporter vector (Promega) in which luciferase coding sequences were driven by a minimal SV40 promoter, and designated as pGL2-p-GCbox-1/2 wt and pGL2-GCbox-1/2 mut. HEK293 cells were seeded in the 48-well plate. After overnight incubation, cells were co-transfected with $100 \mathrm{ng}$ of firefly luciferase reporter plasmid, $4 \mathrm{ng}$ of pSV-Renilla plasmid, and the indicated plasmids followed by culturing in nor- mal or serum-free medium. Luciferase activities were measured using the Dual-Luciferase Reporter Assay System (Promega). Firefly luciferase activity was normalized to renilla luciferase activity.

\section{ChIP assay}

ChIP assay was performed with an EZ-ChIP kit (Millipore) following the manufacturer's instruction. Briefly, cells were sonicated by Bioruptor Sonication System UCD-300. DNA was immunoprecipitated by Rabbit IgG or SP1 (Proteintech) antibody, followed by qRT-PCR analysis using the following primers: SP1 GCbox-1-forward: 5'-TGAGGCGCTGAGAGGAACTT-3', GCbox-1-reverse: 5'-TCGACAGCGCGTCGATGACGT-3'; GCbox-2 forward: 5'-CACTTCTTTTAAAAGCAGCAGCC-3', GCbox-2 reverse: 5'-TGAGGCAGGAGGAGGCTGC-3'.

\section{Fluorescent confocal microscopy}

Fluorescent confocal microscope (ZEISS710) and ZEN microscope imaging software (ZEISS) were used to acquire and analyze the images. The percentage of cells with punctate GFP-LC3 fluorescence was calculated.

\section{Animal studies}

All animal studies were conducted with approval from the Animal Research Ethics Committee of the University of Science and Technology of China. For xenograft experiment, each group of HepG2 cells $\left(5 \times 10^{6}\right)$ was injected subcutaneously into nude mice (SJA Laboratory Animal Company). For ketone body injection experiment, mice were administered with either saline alone or saline containing $\beta$-HB $(500 \mathrm{mg} / \mathrm{kg})$ by daily i.p. injections. For ketogenic diet experiment, mice were fed under ketogenic diet (KD) or normal diet (ND) condition. The ingredients of the ketogenic diets were shown in Supplementary information, Table S6. Mouse body weight, blood glucose and $\beta$-HB levels were monitored during the experiments. Mouse blood was obtained from tail clips and blood glucose and $\beta$-HB were measured using a Free Style Optium Blood Glucose and Ketone Monitoring System (Abbott Diabetes Care Ltd., UK). Tumor volumes were calculated using the following formula: width $(\mathrm{mm}) \times$ depth $(\mathrm{mm}) \times$ length $(\mathrm{mm}) \times 0.52$.

\section{Measurement of serum $\beta$-HB in normal subjects and HCC patients}

The serum samples of 29 normal subjects and 35 HCC patients were collected by the Department of General Surgery, Anhui Provincial Hospital. To use these clinical materials for research purposes, prior patients' written informed consents and approval from the Institutional Research Ethics Committee of Anhui Provincial Hospital were obtained (Approval No. 201367). Serum $\beta$-HB was measured by $\beta$-HB Assay Kit (Cayman).

\section{Clinical human tissue specimen}

The normal liver tissues were collected from patients undergoing resection of hepatic hemangiomas at the Department of Hepatobiliary Surgery, the First Affiliated Hospital of Sun Yat-sen University. Formalin-fixed, paraffin-embedded primary HCC specimens obtained from 158 patients were randomly selected from the archives of the Sun Yat-sen University Cancer Center (Guangzhou, China). The 20 paired HCC lesions and the adjacent noncancerous clinical tissue samples were collected from HCC patients. To 
use these clinical materials for research purposes, prior patients' written informed consents and approval from the Institutional Research Ethics Committee of Sun Yat-sen University Cancer Center were obtained. Tumor clinical stages were defined according to the 2002 American Joint Committee on Cancer/International Union against Cancer tumor/lymph node metastasis/distal metastasis (TNM) classification system [60].

\section{IHC}

IHC staining was quantitatively analyzed with the AxioVision Rel.4.6 computerized image analysis system assisted with the automatic measurement program (Carl Zeiss, Oberkochen, Germany). Ten representative staining fields of each section were analyzed to verify the Mean Optical Density (MOD). The MOD data were statistically analyzed by $t$-test to compare the average MOD difference between different groups of tissues.

\section{Statistical analysis}

The relationship between OXCT1 expression and clinicopathological characteristics was analyzed by the $X^{2}$ test. Survival curves were plotted by the Kaplan-Meier method and compared using the log-rank test. Data are presented as the mean $\pm \mathrm{SD}$ of at least three independent experiments. Statistical significance $(P<0.05)$ was assessed by the Student's $t$-test unless otherwise noted.

\section{Acknowledgments}

Our work is supported in part by the National Basic Key Research Program of China (2014CB910600 and 2012CB910104), the National Natural Science Foundation of China (31371429, 31571472, 81372148, 81530076 and 81525022), Chinese Academy of Sciences (XDA01010404), the Fundamental Research Funds for the Central Universities, the Development Foundation of Hefei Center for Physical Science and Technology, and CAS Key laboratory of Innate Immunity and Chronic Disease.

\section{Author Contributions}

$\mathrm{H}$ Zhang and $\mathrm{P}$ Gao conceived this study. D Huang, H Zhang, P Gao, CV Dang, S Lin, L Song and W Jia designed the experiments. D Huang, T Li, L Wang, L Zhang, R Yan, K Li, G Wu, X Song and $\mathrm{L} \mathrm{Hu}$ performed the experiments. L Song organized the analysis of clinical HCC samples. W Jia provided the serum specimen of HCC patients and normal subjects. H Zhang, P Gao and D Huang wrote the paper. All the authors read and approved the manuscript.

\section{Competing Financial Interests}

The authors declare no competing financial interests.

\section{References}

1 Boroughs LK, DeBerardinis RJ. Metabolic pathways promoting cancer cell survival and growth. Nat Cell Biol 2015; 17:351-359.

2 Vander Heiden MG, Cantley LC, Thompson CB. Understanding the Warburg effect: the metabolic requirements of cell proliferation. Science 2009; 324:1029-1033.

3 DeNicola GM, Cantley LC. Cancer's fuel choice: new flavors for a picky rater. Mol Cell 2015; 60:514-523.
4 Comerford SA, Huang Z, Du X, et al. Acetate dependence of tumors. Cell 2014; 159:1591-1602.

5 Lyssiotis CA, Cantley LC. Acetate fuels the cancer engine. Cell 2014; 159:1492-1494.

6 Mashimo T, Pichumani K, Vemireddy V, et al. Acetate is a bioenergetic substrate for human glioblastoma and brain metastases. Cell 2014; 159:1603-1614.

7 Loo JM, Scherl A, Nguyen A, et al. Extracellular metabolic energetics can promote cancer progression. Cell 2015; 160:393-406.

8 Doherty JR, Cleveland JL. Targeting lactate metabolism for cancer therapeutics. J Clin Invest 2013; 123:3685-3692.

9 Young VR. Energy metabolism and requirements in the cancer patient. Cancer Res 1977; 37:2336-2347.

10 Lee DC, Sohn HA, Park ZY, et al. A lactate-induced response to hypoxia. Cell 2015; 161:595-609.

11 Newman JC, Verdin E. Ketone bodies as signaling metabolites. Trends Endocrinol Metab 2014; 25:42-52.

12 Owen OE, Morgan AP, Kemp HG, Sullivan JM, Herrera MG, Cahill GF. Brain metabolism during fasting. J Clin Invest 1967; 46:1589-1595.

13 Fukao T, Lopaschuk GD, Mitchell GA. Pathways and control of ketone body metabolism: on the fringe of lipid biochemistry. Prostaglandins Leukot Essent Fatty Acids 2004; 70:243251.

14 Fukao T, Song XQ, Mitchell GA, et al. Enzymes of ketone body utilization in human tissues: Protein and messenger RNA levels of succinyl-coenzyme A (CoA):3-ketoacid CoA transferase and mitochondrial and cytosolic acetoacetyl-CoA thiolases. Pediatr Res 1997; 42:498-502.

15 Orii KE, Fukao T, Song XQ, Mitchell GA, Kondo N. Liver-specific silencing of the human gene encoding succinyl-CoA:3-ketoacid CoA transferase. Tohoku J Exp Med 2008; 215:227-236.

16 Huang D, Li TT, Li XH, et al. HIF-1-mediated suppression of ccyl-CoA dehydrogenases and fatty acid oxidation is critical for cancer progression. Cell Rep 2014; 8:1930-1942.

17 Ma X, Li C, Sun L, et al. Lin28/let-7 axis regulates aerobic glycolysis and cancer progression via PDK1. Nat Commun 2014; 5:5212.

18 Sun LC, Song LB, Wan QF, et al. cMyc-mediated activation of serine biosynthesis pathway is critical for cancer progression under nutrient deprivation conditions. Cell Res 2015; 25:429-444.

19 Thorrez L, Laudadio I, Van Deun K, et al. Tissue-specific disallowance of housekeeping genes: The other face of cell differentiation. Genome Res 2011; 21:95-105.

20 Cahill GF. Fuel metabolism in starvation. Annu Rev Nutr 2006; 26:1-22.

21 Wolfrum C, Asilmaz E, Luca E, Friedman JM, Stoffel M. Foxa2 regulates lipid metabolism and ketogenesis in the liver during fasting and in diabetes. Nature 2004; 432:1027-1032.

22 Pfeifer AMA, Cole KE, Smoot DT, et al. Simian virus-40 large tumor antigen-immortalized normal human liver epithelial-cells express hepatocyte characteristics and metabolize chemical carcinogens. Proc Natl Acad Sci USA 1993; 90:5123-5127.

23 Girard J, Ferre P, Pegorier JP, Duee PH. Adaptations of glucose and fatty acid metabolism during perinatal period and 
suckling-weaning transition. Physiol Rev 1992; 72:507-562.

24 Levin VA, Panchabhai SC, Shen L, Kornblau SM, Qiu YH, Baggerly KA. Different changes in protein and phosphoprotein levels result from serum starvation of high-grade glioma and adenocarcinoma cell lines. J Proteome Res 2010; 9:179191.

25 Pirkmajer S, Chibalin AV. Serum starvation: caveat emptor. Am J Physiol Cell Physiol 2011; 301:C272-C279.

26 Sancho P, Fabregat I. NADPH oxidase NOX1 controls autocrine growth of liver tumor cells through up-regulation of the epidermal growth factor receptor pathway. J Biol Chem 2010; 285:24815-24824.

27 Jung YD, Nakano K, Liu WB, Gallick GE, Ellis LM. Extracellular signal-regulated kinase activation is required for up-regulation of vascular endothelial growth factor by serum starvation in human colon carcinoma cells. Cancer Res 1999; 59:4804-4807.

28 Grimm S, Bauer MK, Baeuerle PA, Schulze-Osthoff K. Bcl-2 down-regulates the activity of transcription factor NF-kappaB induced upon apoptosis. J Cell Biol 1996; 134:13-23.

29 Jacinto E, Facchinetti V, Liu D, et al. SIN1/MIP1 maintains rictor-mTOR complex integrity and regulates Akt phosphorylation and substrate specificity. Cell 2006; 127:125-137.

30 Chu S, Ferro TJ. Sp1: regulation of gene expression by phosphorylation. Gene 2005; 348:1-11.

31 Lin SY, Li TY, Liu Q, et al. GSK3-TIP60-ULK1 signaling pathway links growth factor deprivation to autophagy. Science 2012; 336:477-481.

32 Lum JJ, Bauer DE, Kong M, et al. Growth factor regulation of autophagy and cell survival in the absence of apoptosis. Cell 2005; 120:237-248.

33 Kondo Y, Kanzawa T, Sawaya R, Kondo S. The role of autophagy in cancer development and response to therapy. Nat Rev Cancer 2005; 5:726-734.

34 Shimizu S, Kanaseki T, Mizushima N, et al. Role of Bcl-2 family proteins in a non-apoptotic programmed cell death dependent on autophagy genes. Nat Cell Biol 2004; 6:1221-1228.

35 Hardie DG. AMPK-sensing energy while talking to other signaling pathways. Cell Metab 2014; 20:939-952.

36 Zhang CS, Jiang B, Li MQ, et al. The lysosomal v-ATPase-regulator complex is a common activator for AMPK and mTORC1, acting as a switch between catabolism and anabolism. Cell Metab 2014; 20:526-540.

37 Kim J, Kundu M, Viollet B, Guan KL. AMPK and mTOR regulate autophagy through direct phosphorylation of Ulk1. Nat Cell Biol 2011; 13:132-141.

38 Allen BG, Bhatia SK, Buatti JM, et al. Ketogenic diets enhance oxidative stress and radio-chemo-therapy responses in lung cancer xenografts. Clin Cancer Res 2013; 19:3905-3913.

39 Dang MT, Wehrli S, Dang CV, Curran T. The ketogenic diet does not affect growth of hedgehog pathway medulloblastoma in mice. PLos One 2015; 10:e 0133633.

40 Locasale JW. Serine, glycine and one-carbon units: cancer metabolism in full circle. Nat Rev Cancer 2013; 13:572-583.

41 Cahill GF Jr, Veech RL. Ketoacids? Good medicine? Trans Am Clin Climatol Assoc 2003; 114:149-161.

42 Rabinowitz JD, White E. Autophagy and metabolism. Science
2010; 330:1344-1348.

43 Russell RC, Yuan HX, Guan KL. Autophagy regulation by nutrient signaling. Cell Res 2014; 24:42-57.

44 Gunn JM, Clark MG, Knowles SE, Hopgood MF, Ballard FJ. Reduced rates of proteolysis in transformed-cells. Nature 1977; 266:58-60.

45 Gozuacik D, Kimchi A. Autophagy as a cell death and tumor suppressor mechanism. Oncogene 2004; 23:2891-2906.

$46 \mathrm{Qu}$ XP, Yu J, Bhagat G, et al. Promotion of tumorigenesis by heterozygous disruption of the beclin 1 autophagy gene. $J$ Clin Invest 2003; 112:1809-1820.

47 Buchser WJ, Laskow TC, Pavlik PJ, Lin HM, Lotze MT. Cell-mediated autophagy promotes cancer cell survival. Cancer Res 2012; 72:2970-2979.

48 Yang SH, Wang XX, Contino G, et al. Pancreatic cancers require autophagy for tumor growth. Gene Dev 2011; 25:717729.

49 Shimazu T, Hirschey MD, Newman J, et al. Suppression of oxidative stress by beta-hydroxybutyrate, an endogenous histone deacetylase inhibitor. Science 2013; 339:211-214.

50 Taggart AKP, Kero J, Gan XD, et al. (D)-beta-hydroxybutyrate inhibits adipocyte lipolysis via the nicotinic acid receptor PUMA-G. J Biol Chem 2005; 280:26649-26652.

51 Chen JS, Wang Q, Fu XH, et al. Involvement of PI3K/PTEN/ AKT/mTOR pathway in invasion and metastasis in hepatocellular carcinoma: association with MMP-9. Hepatol Res 2009; 39:177-186.

52 Franke TF, Yang SI, Chan TO, et al. The protein kinase encoded by the Akt proto-oncogene is a target of the PDGF-activated phosphatidylinositol 3-kinase. Cell 1995; 81:727-736.

53 Ching JK, Rajguru P, Marupudi N, Banerjee S, Fisher JS. A role for AMPK in increased insulin action after serum starvation. Am J Physiol Cell Physiol 2010; 299:C1171-C1179.

54 Lum JJ, DeBerardinis RJ, Thompson CB. Autophagy in metazoans: cell survival in the land of plenty. Nat Rev Mol Cell Bio 2005; 6:439-448.

55 Hahn-Windgassen A, Nogueira V, Chen CC, Skeen JE, Sonenberg N, Hay N. Akt activates the mammalian target of rapamycin by regulating cellular ATP level and AMPK activity. J Biol Chem 2005; 280:32081-32089.

56 Carey BW, Finley LW, Cross JR, Allis CD, Thompson CB. Intracellular alpha-ketoglutarate maintains the pluripotency of embryonic stem cells. Nature 2015; 518:413-416.

57 Fan TW, Lane AN, Higashi RM, Yan J. Stable isotope resolved metabolomics of lung cancer in a SCID mouse model. Metabolomics 2011; 7:257-269.

58 Millard P, Letisse F, Sokol S, Portais JC. IsoCor: correcting MS data in isotope labeling experiments. Bioinformatics 2012; 28:1294-1296.

59 Zhang HF, Gao P, Fukuda R, et al. HIF-1 inhibits mitochondrial biogenesis and cellular respiration in VHL-deficient renal cell carcinoma by repression of C-MYC activity. Cancer Cell 2007; 11:407-420.

60 Xie C, Wu JH, Yun JP, et al. MACC1 as a prognostic biomarker for early-stage and AFP-normal hepatocellular carcinoma. PLos One 2013; 8:e64235.

(Supplementary information is linked to the online version of the paper on the Cell Research website.) 